\title{
Spatial Degrees of Freedom in Everett Quantum Mechanics*
}

\author{
Mark A. Rubin \\ Lincoln Laboratory \\ Massachusetts Institute of Technology \\ 244 Wood Street \\ Lexington, Massachusetts 02420-9185 \\ rubin@LL.mit.edu
}

\begin{abstract}
Stapp claims that, when spatial degrees of freedom are taken into account, Everett quantum mechanics is ambiguous due to a "core basis problem." To examine an aspect of this claim I generalize the ideal measurement model to include translational degrees of freedom for both the measured system and the measuring apparatus. Analysis of this generalized model using the Everett interpretation in the Heisenberg picture shows that it makes unambiguous predictions for the possible results of measurements and their respective probabilities. The presence of translational degrees of freedom for the measuring apparatus affects the probabilities of measurement outcomes in the same way that a mixed state for the measured system would. Examination of a measurement scenario involving several observers illustrates the consistency of the model with perceived spatial localization of the measuring apparatus.
\end{abstract}

Key words: Everett interpretation, quantum mechanics, basis problem, Heisenberg picture

\section{Introduction}

\subsection{Aim of the Present Paper}

The idea that the Everett interpretation ${ }^{(1-4)}$ of quantum mechanics contains an intrinsic ambiguity regarding the description of the measurement process has been present in the

${ }^{*}$ This work was sponsored by the Air Force under Air Force Contract FA8721-05-C-0002. Opinions, interpretations, conclusions, and recommendations are those of the author and are not necessarily endorsed by the U.S. Government. 
literature for decades. ${ }^{(5-9)}$ This notion, variously referred to as the "basis ambiguity," the "preferred basis problem," or simply the "basis problem," is generally presented in the context of models of measuring devices and measured systems with discrete degrees of freedom. In the context of such models I have recently given an explicit demonstration, $\frac{(10)}{10}$ following the approach of DeWitt, 11 12) that there is no basis problem; i.e., that the Everett-interpretation description of measurement is in fact unambiguous.

All real-world physical systems contain, however, not just discrete degrees of freedom, but also continuous spatial (translational and rotational) degrees of freedom. In this paper I extend the analysis of the purely-discrete case $\underline{(10)}$ with this fact in mind. Specifically, I present a model of measurement situations in which both the measurement apparatus and the observed system possess translational degrees of freedom, and show that, despite this extension, the Everett description of measurement remains unambiguous. As in the purely-discrete case, the model is one of isolated measurements; i.e., no role is played by decoherence (see Ref. 13 and references therein).

\subsection{Stapp's Core Basis Problem}

Stapp ${ }^{(14)}$ claims that, in systems with spatial degrees of freedom such as those considered in the present paper, the basis problem may be present in aggravated form. Quantum theory must satisfy the following two requirements:

Outcome determination. The formalism must unambiguously determine which specific outcomes observers will perceive to occur in a measurement situation, as well as the respective associated probabilities. The outcomes must form a discrete set, since "the normal rules for extracting well defined probabilities from a quantum state require the specification, or singling out, of a discrete set (i.e., a denumerable set) of orthogonal subspaces, one for each of a set of alternative possible experientially distinguishable observations." (14)

Localization. The predictions of the formalism must be consistent with observers' perception that measuring devices have well-defined spatial locations.

Stapp argues that Everett quantum mechanics fails to satisfy these requirements, and terms this failure the "core basis problem:"

The essential point is that if the universe has been evolving since the big bang in accordance with the Schrödinger equation, then it must by now be an amorphous structure in which every device is a smeared-out cloud of a continuum of different possibilities... . Due to the uncertainty principle, each particle would have had a tendency to spread out. Thus various particles with various momenta would have been able to combine and condense in myriads of ways into bound structures, including measuring devices, whose centers, orientations, and fine details would necessarily be smeared out over continua of possibilities... But how can a particular discrete set of orthogonal subspaces be picked out from an amorphous continuum by the action of the Schrödinger equation alone?... [The problem is that of] specifying a discrete basis for the probability computations associated with our apparently discrete (Yes or No) experiences, when the Schrödinger equation generates continuous evolution of an ever-amorphous state. $\frac{(14)}{(1)}$ 
Stapp also argues that decoherence, which is sometimes invoked to allow Everett quantum mechanics to satisfy the outcome-determination requirement, can only do so at the expense of producing a theory violating the localization requirement. Since, as mentioned above, the model presented here does not make use of decoherence, I will not address this specific issue.

Rather, I will show directly, for the case of a simple model of measurements with translational degrees of freedom but without decoherence, that Everett quantum mechanics does in fact satisfy both the outcome-determination and localization requirements.

\subsection{Everett Quantum Mechanics in the Heisenberg Picture}

It proves convenient to perform the analysis working in the Heisenberg picture. In this picture, the properties of physical objects at time $t$ are determined jointly by time-dependent operators and a time-independent state vector which encodes initial-condition information specified at an initial time $t_{i n}<t .{ }^{1}$ So, interpretation of the formalism cannot be based, as it is in the Schrödinger picture, upon "branching" of the state vector during the course of the measurement interaction - the Heisenberg-picture state vector does not "branch" or in any other way change its form. Rather, the operator which represents the degree of freedom of the measuring device relevant for its role as a measuring device evolves from a form which represents a "ready state" or "state of ignorance" to a characteristic form which indicates that the degree of freedom in question has split into Everett copies, each of which possesses a definite value. (Measurement in Heisenberg-picture Everett quantum mechanics has been described in Refs. 15, 16, and 10, and is reviewed in Sec. 2 below.)

The possible outcomes for a measurement depend on the spectrum of the operator representing that particular degree of freedom. If the spectrum of this operator is discrete, the possible outcomes will be distinct. So, only operators with discrete spectra can correspond to measurement results/states of awareness. Such operators will be present in systems of finite size; e.g., the operators corresponding to the energy associated with relative motion of bound constituents, and functions of these operators.

Probabilities can be assigned to these distinct outcomes using the same rules which would apply were the discrete degrees of freedom the only ones present, since these rules make reference only to the operator representing the outcome, not to any other operators pertaining to the measuring apparatus (such as its position in space). As far as measurement outcomes recorded/perceived by a measuring device/observer are concerned, the spatial degrees of freedom are simply "along for the ride."

One may object that translational degrees of freedom are measured all the time, and that it must be possible to assign them outcomes and probabilities in a consistent manner. However, any measurement of such a continuous variable is performed by some finite-size discrete-spectrum measuring device, and ultimately perceived by a finite-size discrete-spectrum brain. So, even when measuring a continuous degree of freedom, the measuring/observing system splits into Everett copies corresponding to discrete outcomes.

What about spatial localization of macroscopic objects such as measuring devices and

\footnotetext{
${ }^{1}$ The specific choice for $t_{i n}$ is arbitrary, although in any given situation some choices for $t_{\text {in }}$ may be more convenient than others.
} 
brains? Does such an object indeed become, in the Everett interpretation, a "smeared-out cloud of a continuum of different possibilities?"

In the first-quantized Heisenberg picture which we are employing here, the operator representing a discrete "internal" degree of freedom, corresponding to the value obtained by a measuring device, does not have a spatial location. Position, in the first-quantized formalism, is an operator, and so cannot parameterize another operator. ${ }^{2}$ Therefore, localization must be demonstrated operationally; that is, by computing the results of measurements performed by several measuring devices, and showing that the results are consistent with the perception of definite positions for the measuring devices. An example of this procedure will be presented in Sec. 4 below. The upshot is that, provided the measurement interactions are localized in space, the results of measurements are consistent with perceived spatial localization of the devices which perform the measurements.

\subsection{Organization of the Paper}

Sec. 2 reviews the formalism of ideal measurements in Heisenberg-picture Everett quantum mechanics. Sec. 3 introduces a model for finite-range interactions of measured systems and measuring devices with translational degrees of freedom, analyzes this model in the context of the Heisenberg-picture Everett interpretation, and shows that it makes unambiguous predictions for the possible outcomes of measurements and their respective probabilities. The translational degrees of freedom of the measuring device, usually ignored, are shown to have the same effect on probabilities of measurement outcomes as would an initial mixed state for the measured system. Sec. 4 analyzes a measurement situation involving more than one observer, showing that the formalism is consistent with the notion of measuring devices having well-defined spatial locations. These results are summarized and discussed in Sec. 5. The Appendix extends the operator expansion uniqueness theorem of Ref. 10 to systems with continuous degrees of freedom.

\section{Heisenberg-Picture Everett Quantum Theory with Discrete Degrees of Freedom}

The basic model for a measurement situation in quantum mechanics, so-called "ideal measurement" (see, e.g., Ch. 14 of Ref. 18), involves a physical system with a degree of freedom $\mathcal{S}$ the properties of which are measured by an observer with a degree of freedom $\mathcal{O}$. These will generally be referred to simply as "the system" and "the observer" respectively. (The use of the term "observer" is not meant to imply that $\mathcal{O}$ is necessarily a degree of freedom of a sentient system; e.g., $\mathcal{O}$ might be a degree of freedom of a piece of laboratory apparatus.) The respective state spaces of $\mathcal{S}$ and $\mathcal{O}$ are spanned by the vectors

$$
\left|\mathcal{S} ; \alpha_{i}\right\rangle, \quad i=1, \ldots, M
$$

\footnotetext{
${ }^{2}$ In Ref. 17 I employ the field-theoretic Heisenberg-picture formalism, in which spatial position is a c-number and parameterizes operator-valued fields, to show the locality of information flow in entangled second-quantized systems. However, only the measured systems are explicitly represented by field operators there, not the measuring instruments.
} 


$$
\left|\mathcal{O} ; \beta_{i}\right\rangle, \quad i=0, \ldots, M,
$$

where the labels $\alpha_{i}$ and $\beta_{i}$ are, respectively, the nondegenerate eigenvalues of Hermitian time-independent (i.e., Schrödinger-picture) operators $\widehat{a}$ and $\widehat{b}$ :

$$
\begin{gathered}
\widehat{a}\left|\mathcal{S} ; \alpha_{i}\right\rangle=\alpha_{i}\left|\mathcal{S}: \alpha_{i}\right\rangle, \quad i=1, \ldots, M, \\
\alpha_{i}=\alpha_{j} \Rightarrow i=j, \quad i, j=1, \ldots, M, \\
\widehat{b}\left|\mathcal{O} ; \beta_{i}\right\rangle=\beta_{i}\left|\mathcal{O} ; \beta_{i}\right\rangle, \quad i=0, \ldots, M, \\
\beta_{i}=\beta_{j} \Rightarrow i=j, \quad i, j=0, \ldots, M .
\end{gathered}
$$

The vectors (11), (2) are taken to be normalized and so, from (3)-(16), are orthonormal:

$$
\begin{aligned}
& \left\langle\mathcal{S} ; \alpha_{i} \mid \mathcal{S} ; \alpha_{j}\right\rangle=\delta_{i j}, \quad i, j=1, \ldots, M, \\
& \left\langle\mathcal{O} ; \beta_{i} \mid \mathcal{O} ; \beta_{j}\right\rangle=\delta_{i j}, \quad i, j=0, \ldots, M .
\end{aligned}
$$

The measurement is a physical interaction between $\mathcal{S}$ and $\mathcal{O}$. Prior to the measurement the combined state of $\mathcal{S}$ and $\mathcal{O}$ is a product state, implying that the respective observables of $\mathcal{S}$ and $\mathcal{O}$ are uncorrelated. In addition, $\mathcal{O}$ is taken to be in the "ignorant" or "ready" state $\left|\mathcal{O} ; \beta_{0}\right\rangle$ while the state of $\mathcal{S}$ is arbitrary. It is convenient to take the time immediately prior to the measurement to be the time $t_{i n}$ at which the time-independent Heisenberg-picture state vector $\left|\psi\left(t_{i n}\right)\right\rangle$ is defined. That is, $t_{i n}$ is the time at which the Heisenberg-picture state vector is equal to the Schrödinger-picture state vector. Then

$$
\left|\psi\left(t_{\text {in }}\right)\right\rangle=\left|\mathcal{O} ; \beta_{0}\right\rangle|\mathcal{S} ; \psi\rangle
$$

where

$$
\begin{gathered}
|\mathcal{S} ; \psi\rangle=\sum_{i=1}^{M} \psi_{i}\left|\mathcal{S} ; \alpha_{i}\right\rangle, \\
\sum_{i=1}^{M}\left|\psi_{i}\right|^{2}=1, \quad\left\{\psi_{i}\right\} \text { otherwise arbitrary. }
\end{gathered}
$$

Between time $t_{i n}$ and time $t$, any operator $\widehat{o}$ evolves according to the Heisenberg-picture dynamical rule

$$
\widehat{o}(t)=\widehat{U}_{I}^{\dagger} \widehat{o}\left(t_{i n}\right) \widehat{U}_{I}
$$

where $\widehat{U}_{I}$ is the unitary time-evolution operator,

$$
\widehat{U}_{I}=\exp \left(-i \widehat{H}_{I}\left(t-t_{i n}\right)\right)
$$

We will generally drop the time argument in Heisenberg-picture operators evaluated at the initial time $t_{i n}$, since they are then just equal to their Schrödinger-picture counterparts:

$$
\widehat{o}\left(t_{i n}\right)=\widehat{o}
$$

For an ideal measurement, the Hamiltonian $\widehat{H}_{I}$ has the form

$$
\widehat{H}_{I}=\sum_{i=1}^{M} \widehat{h}_{i}^{\mathcal{O}} \otimes \widehat{P}_{i}^{\mathcal{S}} .
$$


Here $\widehat{h}_{i}^{\mathcal{O}}$ acts only in the space of $\mathcal{O}$, and $\widehat{P}_{i}^{\mathcal{S}}$ is the projection operator into the $i^{\text {th }}$ to-bemeasured state of $\mathcal{S}$ :

$$
\widehat{P}_{i}^{\mathcal{S}}=\left|\mathcal{S} ; \alpha_{i}\right\rangle\left\langle\mathcal{S} ; \alpha_{i}\right|, \quad i=1, \ldots, M
$$

satisfying

$$
\begin{gathered}
\widehat{P}_{i}^{\mathcal{S}} \widehat{P}_{j}^{\mathcal{S}}=\delta_{i j} \widehat{P}_{j}^{\mathcal{S}}, \\
\sum_{i=1}^{M} \widehat{P}_{i}^{\mathcal{S}}=1 .
\end{gathered}
$$

Using (71), (15) and (16) in (13),

$$
\widehat{U}_{I}=\sum_{i=1}^{M} \widehat{u}_{i}^{\mathcal{O}} \otimes \widehat{P}_{i}^{\mathcal{S}}
$$

where

$$
\widehat{u}_{i}^{\mathcal{O}}=\exp \left(-i \widehat{h}_{i}^{\mathcal{O}}\left(t-t_{i n}\right)\right), \quad i=1, \ldots, M .
$$

For an ideal measurement, the $\widehat{u}_{i}^{\mathcal{O}}$ must have the property that

$$
\widehat{u}_{i}^{\mathcal{O}}\left|\mathcal{O} ; \beta_{0}\right\rangle=\left|\mathcal{O} ; \beta_{i}\right\rangle, \quad i=1, \ldots, M .
$$

E.g., if the $\widehat{h}_{i}^{\mathcal{O}}$ 's are of the form

$$
\widehat{h}_{i}^{\mathcal{O}}=i \kappa\left(\left|\mathcal{O} ; \beta_{i}\right\rangle\left\langle\mathcal{O} ; \beta_{0}|-| \mathcal{O} ; \beta_{0}\right\rangle\left\langle\mathcal{O} ; \beta_{i}\right|\right), \quad i=1, \ldots, M
$$

then (21) will be satisfied provided

$$
\kappa=\frac{\pi}{2\left(t-t_{\text {in }}\right)}
$$

From (7), (12), (16) and (19) it follows that

$$
\widehat{b}(t)=\sum_{i=1}^{M} \widehat{b}_{i} \otimes \widehat{P}_{i}^{\mathcal{S}}
$$

where

$$
\widehat{b}_{i}=\widehat{u}_{i}^{\mathcal{O}} \widehat{b}_{u_{i}^{\mathcal{O}}}, \quad i=1, \ldots, M
$$

From (15), (21) and (25),

$$
\widehat{b}_{i}\left|\mathcal{O} ; \beta_{0}\right\rangle=\beta_{i}\left|\mathcal{O} ; \beta_{0}\right\rangle \quad i=1, \ldots, M \text {. }
$$

The fact that $\widehat{b}(t)$ has the form (24) and satisfies (26) with the initial state of the form (9) is taken to indicate that the physical degree of freedom $\mathcal{O}$ described by $\widehat{b}(t)$ has been split into $M$ Everett copies with respective values $\beta_{1}, \ldots, \beta_{M}$. This is termed "interpretive rule 1." (16)

As has been shown in Sec. 4.2.1 of Ref. 10, the decomposition (24) satisfying (26) is unique. I.e., there is no inequivalent decomposition of $\widehat{b}(t)$ of the form (24) satisfying (26) 
which would correspond to the observer having measured some different property of the system. There is thus no "basis ambiguity."

The projectors $\widehat{P}_{i}^{\mathcal{S}}$ which multiply the $\widehat{b}_{i}$ 's serve as "labels" (15) attached to the Everett copies, carrying information about past interactions and playing an important in role in the Everett-interpretation resolution of the EPR paradox. 190 [20] 17)

Taken by itself, interpretive rule 1 would have us conclude that all physically-possible outcomes to a measurement are realized every time that measurement is performed, even when the amplitudes $\psi_{i}$ for one or more outcomes are equal to zero. To enable the formalism to be applicable to situations in which not all possible outcomes are realized, interpretive rule 1 must be supplemented ${ }^{(16)}$ by interpretive rule 2: Only those copies of $\mathcal{O}$ exist at time $t$ which satisfy $W_{i}(t) \neq 0$, where the "weight" $W_{i}(t)$ associated with the $i^{\text {th }}$ copy is the matrix element of the label between the initial-state bra and ket:

$$
W_{i}(t)=\left\langle\psi\left(t_{i n}\right)\left|\widehat{P}_{i}^{\mathcal{S}}\right| \psi\left(t_{i n}\right)\right\rangle .
$$

By introducing an ensemble of systems identical to $\mathcal{S}$ and accompanied by their respective observers $\mathcal{O}$, one can, using interpretive rules 1 and 2 and physically-based restrictions on the properties of measurement devices (finite resolution and information-storage capacity), derive ${ }^{(16)}$ both the existence of probability in quantum mechanics and the Born rule for the value of probability, where probability is in the sense of the frequency interpretation, i.e, relative frequency in the limit of an infinitely-large ensemble(see, e.g., Refs. 21 and 22.) The use of the Heisenberg picture avoids problems associated with other approaches to deriving frequency-interpretation probability in the Everett interpretation (see Ref. 16 for details and references). The value of the probability associated with the $i^{\text {th }}$ outcome of the ideal measurement interaction is $W_{i}(t)$; using (18) in (27), we see that

$$
\sum_{i=1}^{M} W_{i}(t)=\left\langle\psi\left(t_{i n}\right)\left|\sum_{i=1}^{M} \widehat{P}_{i}^{\mathcal{S}}\right| \psi\left(t_{i n}\right)\right\rangle=1 .
$$

\section{$3 \quad$ Heisenberg-Picture Everett Quantum Theory with Translational Degrees of Freedom}

\subsection{A Localized Measurement Interaction Model}

\subsubsection{State Spaces}

To investigate the role of spatial degrees of freedom, we must extend the ideal-measurement model. We first introduce translational degrees of freedom $\mathcal{X}$ and $\mathcal{Z}$ for the system and the observer, respectively. The state space is spanned by tensor products of the discrete sets of basis vectors (11), (2) and the continuous sets of basis vectors

$$
\begin{aligned}
& \left|\mathcal{X}_{i} ; \xi_{i}\right\rangle, \quad-\infty<\xi_{i}<\infty, \quad i=1,2,3, \\
& \left|\mathcal{Z}_{i} ; \zeta_{i}\right\rangle, \quad-\infty<\zeta_{i}<\infty, \quad i=1,2,3,
\end{aligned}
$$

where the labels $\xi_{i}, \zeta_{i}$ are three-dimensional spatial coordinates, eigenvalues of the corresponding position operators:

$$
\widehat{\vec{x}}|\mathcal{X} ; \vec{\xi}\rangle=\vec{\xi}|\mathcal{X} ; \vec{\xi}\rangle
$$




$$
\widehat{\vec{z}}|\mathcal{Z} ; \vec{\zeta}\rangle=\vec{\zeta}|\mathcal{Z} ; \vec{\zeta}\rangle
$$

where

$$
\begin{gathered}
\widehat{\vec{x}}=\left(\widehat{x}_{1}, \widehat{x}_{2}, \widehat{x}_{3}\right), \\
\widehat{\vec{z}}=\left(\widehat{z}_{1}, \widehat{z}_{2}, \widehat{z}_{3}\right), \\
\vec{\xi}=\left(\xi_{1}, \xi_{2}, \xi_{3}\right), \\
\vec{\zeta}=\left(\zeta_{1}, \zeta_{2}, \zeta_{3}\right), \\
|\mathcal{X} ; \vec{\xi}\rangle=\bigotimes_{i=1}^{3}\left|\mathcal{X}_{i} ; \xi_{i}\right\rangle, \\
|\mathcal{Z} ; \vec{\zeta}\rangle=\bigotimes_{i=1}^{3}\left|\mathcal{Z}_{i} ; \zeta_{i}\right\rangle .
\end{gathered}
$$

In terms of the vectors (29), (30) (37) and (38), we can define the $\mathcal{X}$ and $\mathcal{Z}$ projection operators

$$
\begin{aligned}
& \widehat{P}_{\vec{\xi}}^{\mathcal{X}}=\bigotimes_{i=1}^{3} \widehat{P}_{\xi_{i}}^{\mathcal{X}_{i}}=|\mathcal{X} ; \vec{\xi}\rangle\langle\mathcal{X} ; \vec{\xi}| \\
& \widehat{P}_{\vec{\zeta}}^{\mathcal{Z}}=\bigotimes_{i=1}^{3} \widehat{P}_{\zeta_{i}}^{\mathcal{Z}_{i}}=|\mathcal{Z} ; \vec{\zeta}\rangle\langle\mathcal{Z} ; \vec{\zeta}|
\end{aligned}
$$

where

$$
\begin{gathered}
\widehat{P}_{\xi_{i}}^{\mathcal{X}_{i}}=\left|\mathcal{X}_{i} ; \xi_{i}\right\rangle\left\langle\mathcal{X}_{i} ; \xi_{i}\right|, \quad i=1,2,3, \\
\widehat{P}_{\zeta_{i}}^{\mathcal{Z}_{i}}=\left|\mathcal{Z}_{i} ; \zeta_{i}\right\rangle\left\langle\mathcal{Z}_{i} ; \zeta_{i}\right|, \quad i=1,2,3 .
\end{gathered}
$$

In the remainder of this paper we will occasionally use the symbols $\mathcal{S}$ and $\mathcal{O}$ to refer, not just to the discrete degrees of freedom, but to the measured system and observer as a whole, including the spatial degrees of freedom $\mathcal{X}$ and $\mathcal{Z}$. Which meaning is used in any specific case should be clear from the context.

\subsubsection{Measurement Interaction}

We next introduce a spatially-localized measurement interaction. Define the operator

$$
\widehat{P}_{f}^{\mathcal{Z X}}=\int d^{3} \vec{\zeta} d^{3} \vec{\xi} f(\vec{\zeta}, \vec{\xi}) \widehat{P}_{\vec{\zeta}}^{\mathcal{Z}} \widehat{P}_{\vec{\xi}}^{\mathcal{X}}
$$

where $f(\vec{\zeta}, \vec{\xi})$ is a c-number function, and require that $\widehat{P}_{f}^{\mathcal{Z X}}$ be a projection operator,

$$
\left(\widehat{P}_{f}^{\mathcal{Z X}}\right)^{2}=\widehat{P}_{f}^{\mathcal{Z X}}
$$

Using (39)-(43) and imposing the usual normalization conditions for $|\mathcal{X} ; \vec{\xi}\rangle,|\mathcal{Z} ; \vec{\zeta}\rangle$,

$$
\begin{aligned}
& \left\langle\mathcal{X} ; \vec{\xi} \mid \mathcal{X} ; \vec{\xi}^{\prime}\right\rangle=\delta^{3}\left(\vec{\xi}-\vec{\xi}^{\prime}\right), \\
& \left\langle\mathcal{Z} ; \vec{\zeta} \mid \mathcal{Z} ; \vec{\zeta}^{\prime}\right\rangle=\delta^{3}\left(\vec{\zeta}-\vec{\zeta}^{\prime}\right)
\end{aligned}
$$


(44) implies that

$$
(f(\vec{\zeta}, \vec{\xi}))^{2}=f(\vec{\zeta}, \vec{\xi}) \quad \forall \vec{\zeta}, \vec{\xi}
$$

i.e.,

$$
f(\vec{\zeta}, \vec{\xi})=0 \text { or } 1 \quad \forall \vec{\zeta}, \vec{\xi}
$$

To model measurement interactions of finite range, we choose

$$
f(\vec{\zeta}, \vec{\xi})=\theta(a-|\vec{\zeta}-\vec{\xi}|), \quad a>0
$$

where $\theta$ is the Heaviside unit step function. With the choice (49) for $f, \widehat{P}_{f}^{\mathcal{Z} X}$ is the projection operator into the subspace of states in $\mathcal{Z} \otimes \mathcal{X}$ which are within spatial distance $a$ of each other:

$$
\begin{aligned}
\widehat{P}_{f}^{\mathcal{Z X}}|\mathcal{Z} ; \vec{\zeta}\rangle|\mathcal{X} ; \vec{\xi}\rangle & =|\mathcal{Z} ; \vec{\zeta}\rangle|\mathcal{X} ; \vec{\xi}\rangle, & & |\vec{\zeta}-\vec{\xi}|<a \\
& =0, & & |\vec{\zeta}-\vec{\xi}|>a .
\end{aligned}
$$

The finite-range measurement Hamiltonian is taken to be

$$
\widehat{H}_{F}=\widehat{H}_{I} \otimes \widehat{P}_{f}^{\mathcal{Z X}}
$$

where $\widehat{H}_{I}$ is the ideal-measurement Hamiltonian (15). Taking into account (50), the form (51) for $\widehat{H}_{F}$ implies that $\mathcal{S}$ and $\mathcal{O}$ only interact if they are within distance $a$ of each other.

Using (15), (17), (20), (44), and (151), the time-evolution operator

$$
\widehat{U}_{F}=\exp \left(-i \widehat{H}_{F}\left(t-t_{i n}\right)\right)
$$

has the form

$$
\widehat{U}_{F}=\widehat{P}_{\widetilde{f}}^{\mathcal{Z} \mathcal{X}}+\sum_{i=1}^{M} \widehat{u}_{i}^{\mathcal{O}} \otimes \widehat{P}_{i}^{\mathcal{S}} \otimes \widehat{P}_{f}^{\mathcal{Z X}}
$$

Here

$$
\begin{gathered}
\tilde{f}(\vec{\zeta}, \vec{\xi})=1-f(\vec{\zeta}, \vec{\xi}), \\
\widehat{P}_{\widetilde{f}}^{\mathcal{Z}}=\int d^{3} \vec{\zeta} d^{3} \vec{\xi} \tilde{f}(\vec{\zeta}, \vec{\xi}) \widehat{P}_{\vec{\zeta}}^{\mathcal{Z}} \widehat{P}_{\vec{\xi}}^{\mathcal{X}}
\end{gathered}
$$

$\widehat{P} \underset{\widetilde{f}}{\mathcal{X}}$ is the projector into the subspace of states in $\mathcal{Z} \otimes \mathcal{X}$ which are farther apart than $a$ :

$$
\begin{aligned}
\widehat{P} \underset{\widetilde{f}}{\mathcal{Z}}|\mathcal{Z} ; \vec{\zeta}\rangle|\mathcal{X} ; \vec{\xi}\rangle & =|\mathcal{Z} ; \vec{\zeta}\rangle|\mathcal{X} ; \vec{\xi}\rangle, & & |\vec{\zeta}-\vec{\xi}|>a, \\
& =0, & & |\vec{\zeta}-\vec{\xi}|<a .
\end{aligned}
$$

satisfying, from (43), (44), (48), (54) and (55),

$$
\begin{gathered}
\left(\widehat{P}_{\widetilde{f}}^{\mathcal{Z}}\right)^{2}=\widehat{P}_{\widetilde{f}}^{\mathcal{Z X}}, \\
\widehat{P}_{\widetilde{f}}^{\mathcal{Z X}}=1-\widehat{P}_{f}^{\mathcal{Z X}}, \\
\widehat{P}_{\widetilde{f}}^{\mathcal{Z}} \widehat{P}_{f}^{\mathcal{Z X}}=\widehat{P}_{f}^{\mathcal{Z X}} \widehat{P}_{\widetilde{f}}^{\mathcal{Z X}}=0 .
\end{gathered}
$$




\subsubsection{Time Evolution of Operators}

An operator which at time $t_{i n}$ has the value $\widehat{o}$ has, at the later time $t$, the value

$$
\widehat{o}(t)=\widehat{U}_{F}^{\dagger} \widehat{o} \widehat{U}_{F}
$$

If $\widehat{d}=\widehat{d}\left(t_{i n}\right)$ is an operator which at time $t_{\text {in }}$ acts nontrivially only in $\mathcal{O}$-space, then, using (17), (44), (153), (57), (59) and (60),

$$
\widehat{d}(t)=\widehat{d}_{0} \otimes \widehat{P}_{\widetilde{f}}^{\mathcal{Z}}+\sum_{i=1}^{M} \widehat{d}_{i} \otimes \widehat{P}_{i}^{\mathcal{S}} \otimes \widehat{P}_{f}^{\mathcal{Z X}},
$$

where

$$
\begin{gathered}
\widehat{d}_{0}=\widehat{d}, \\
\widehat{d}_{i}=\widehat{u}_{i}^{\mathcal{O} \dagger} \widehat{d} \widehat{u}_{i}^{\mathcal{O}}, \quad i=1, \ldots, M .
\end{gathered}
$$

In particular,

$$
\widehat{b}(t)=\widehat{b}_{0} \otimes \widehat{P}_{\widetilde{f}}^{\mathcal{Z}}+\sum_{i=1}^{M} \widehat{b}_{i} \otimes \widehat{P}_{i}^{\mathcal{S}} \otimes \widehat{P}_{f}^{\mathcal{Z X}}
$$

where

$$
\widehat{b}_{0}=\widehat{b}
$$

and where the $\widehat{b}_{i}$ 's for $i=1, \ldots, M$ are as given in (25). The coefficients of the $\widehat{b}_{i}$ 's in (64) are a complete orthogonal set of projection operators:

$$
\begin{gathered}
\left(\widehat{P}_{\widetilde{f}}^{\mathcal{Z}}\right)^{2}=\widehat{P}_{\widehat{f}}^{\mathcal{Z}}, \\
\widehat{P}_{\widetilde{f}}^{\mathcal{Z}}\left(\widehat{P}_{i}^{\mathcal{S}} \otimes \widehat{P}_{f}^{\mathcal{Z X}}\right)=\left(\widehat{P}_{i}^{\mathcal{S}} \otimes \widehat{P}_{f}^{\mathcal{Z}}\right) \widehat{P}_{\widetilde{f}}^{\mathcal{Z}}=0, \quad i=1, \ldots, M, \\
\left(\widehat{P}_{i}^{\mathcal{S}} \otimes \widehat{P}_{f}^{\mathcal{Z} \mathcal{X}}\right)\left(\widehat{P}_{j}^{\mathcal{S}} \otimes \widehat{P}_{f}^{\mathcal{Z} \mathcal{X}}\right)=\delta_{i j}\left(\widehat{P}_{i}^{\mathcal{S}} \otimes \widehat{P}_{f}^{\mathcal{Z X}}\right), \quad i, j=1, \ldots, M, \\
\widehat{P}_{\widetilde{f}}^{\mathcal{Z} \mathcal{X}}+\sum_{i=1}^{M}\left(\widehat{P}_{i}^{\mathcal{S}} \otimes \widehat{P}_{f}^{\mathcal{Z X}}\right)=1 .
\end{gathered}
$$

As in the completely-discrete case, $\widehat{a}$ is unchanged by the measurement:

$$
\widehat{a}(t)=\widehat{U}_{F}^{\dagger} \widehat{a} \widehat{U}_{F}=\widehat{a} .
$$

The same is true for $\widehat{\vec{x}}$ and $\widehat{\vec{z}}$ :

$$
\begin{aligned}
\widehat{\vec{x}}(t) & =\widehat{U}_{F}^{\dagger} \widehat{\vec{x}} \widehat{U}_{F}=\widehat{\vec{x}} \\
\widehat{\vec{z}}(t) & =\widehat{U}_{F}^{\dagger} \widehat{\vec{z}} \widehat{U}_{F}=\widehat{\vec{z}}
\end{aligned}
$$




\subsubsection{Initial State}

For a measurement situation, the initial state should be one in which the degrees of freedom pertaining to the observer, $\mathcal{O}$ and $\mathcal{Z}$, are uncorrelated with those pertaining to the system to be measured, $\mathcal{S}$ and $\mathcal{X}$. In addition, a measurement of the $\mathcal{O}$ degree of freedom should with certainty determine that the observer is in a state of ignorance. We therefore take the initial state to be

$$
\left|\psi_{1}\left(t_{i n}\right)\right\rangle=\left|\mathcal{Z}, \mathcal{O}, t_{i n}\right\rangle\left|\mathcal{X}, \mathcal{S}, t_{i n}\right\rangle,
$$

where

$$
\begin{gathered}
\left|\mathcal{X}, \mathcal{S}, t_{i n}\right\rangle=\sum_{i=1}^{M} \int d^{3} \vec{\xi} \psi_{i}^{\mathcal{X S}}(\vec{\xi})|\mathcal{X} ; \vec{\xi}\rangle\left|\mathcal{S} ; \alpha_{i}\right\rangle, \\
\left|\mathcal{Z}, \mathcal{O}, t_{i n}\right\rangle=\int d^{3} \vec{\zeta} \psi^{\mathcal{Z}}(\vec{\zeta})|\mathcal{Z} ; \vec{\zeta}\rangle\left|\mathcal{O} ; \beta_{0}\right\rangle
\end{gathered}
$$

and where $\psi_{i}^{\mathcal{X}}(\vec{\xi})$ and $\psi^{\mathcal{Z}}(\vec{\zeta})$ are c-number functions. Normalization of $\left|\mathcal{X}, \mathcal{S}, t_{i n}\right\rangle$ and $\left|\mathcal{Z}, \mathcal{O}, t_{\text {in }}\right\rangle$ imposes the constraints

$$
\begin{gathered}
\sum_{i=1}^{M} \int d^{3} \vec{\xi}\left|\psi_{i}^{\mathcal{X}}(\vec{\xi})\right|^{2}=1, \\
\int d^{3} \vec{\zeta}\left|\psi^{\mathcal{Z}}(\vec{\zeta})\right|^{2}=1 .
\end{gathered}
$$

Using (5), (26) and (65), we see that

$$
\widehat{b}_{i}\left|\mathcal{O} ; \beta_{0}\right\rangle=\beta_{i}\left|\mathcal{O} ; \beta_{0}\right\rangle, \quad i=0, \ldots, M .
$$

So, by virtue of interpretive rule 1 , we conclude from (64), (73) and (78) that at time $t$ the observer, in a state of ignorance at time $t_{i n}$, has split into $M+1$ Everett copies, one of which has remained in a state of ignorance, the others of which have respectively observed the $M$ possible outcomes of successful measurement of $\mathcal{S}$.

Expression (64) is the extension of the expansion (24) to a situation involving translational degrees of freedom. Is there any sort of basis ambiguity present here? Could $\widehat{b}(t)$ also be expanded in a manner different from and physically inequivalent to (64)? The answer to these questions is "no," as may be seen from the generalized version of the operator expansion uniqueness theorem of Sec. 4.2.1 of Ref. 10 that is derived in the Appendix of the present paper.

\subsubsection{Probability}

The probability associated with the Everett copy of $\mathcal{O}$ which remains in a state of ignorance is

$$
W_{b, 0}=\left\langle\psi_{1}\left(t_{i n}\right)\left|\widehat{P}_{\widetilde{f}}^{\mathcal{Z} \mathcal{X}}\right| \psi_{1}\left(t_{i n}\right)\right\rangle .
$$

It should be emphasized that the derivation of "weight equals probability" from relative frequency considerations has only to date been carried out for quantum systems with only discrete degrees of freedom. ${ }^{(16)}$ So, for the present, its application here must be considered 
to be an independent assumption (as is the usual practice in Everett and non-Everett quantum mechanics).

Using (155) and (73)-(75) we find

$$
W_{b, 0}=\sum_{i=1}^{M} \int d^{3} \vec{\zeta} d^{3} \vec{\xi}\left|\psi^{\mathcal{Z}}(\vec{\zeta})\right|^{2} \tilde{f}(\vec{\zeta}, \vec{\xi})\left|\psi_{i}^{\mathcal{X}}(\vec{\xi})\right|^{2}
$$

The probabilities for the other Everett copies are

$$
W_{b, i}=\left\langle\psi_{1}\left(t_{i n}\right)\left|\widehat{P}_{i}^{\mathcal{S}} \otimes \widehat{P}_{f}^{\mathcal{Z X}}\right| \psi_{1}\left(t_{i n}\right)\right\rangle, \quad i=1, \ldots, M .
$$

Using (71), (16), (43) and (73)-(75)

$$
W_{b, i}=\int d^{3} \vec{\zeta} d^{3} \vec{\xi}\left|\psi^{\mathcal{Z}}(\vec{\zeta})\right|^{2} f(\vec{\zeta}, \vec{\xi})\left|\psi_{i}^{\mathcal{X} \mathcal{S}}(\vec{\xi})\right|^{2}, \quad i=1, \ldots, M
$$

Using (18), (76), (77), (80) and (82), we verify that these probabilities properly sum to one:

$$
W_{b, 0}+\sum_{i=1}^{M} W_{b, i}=\int d^{3} \vec{\zeta} d^{3} \vec{\xi}\left|\psi^{\mathcal{Z}}(\vec{\zeta})\right|^{2}(f(\vec{\zeta}, \vec{\xi})+\widetilde{f}(\vec{\zeta}, \vec{\xi})) \sum_{i=1}^{M}\left|\psi_{i}^{\mathcal{X} \mathcal{S}}(\vec{\xi})\right|^{2}=1 .
$$

The Heisenberg-picture Everett formalism is thus seen to provide unambiguous outcome determination, even when the translational degrees of freedom of the observer/measuring device are included in the description of the measurement situation. I.e., the formalism specifies what the outcomes to measurement are -including here the possibility that, due to excessive spatial separation between $\mathcal{S}$ and $\mathcal{O}$, no measurement is made - as well as the respective probabilities of the outcomes, (80) and (82).

\subsection{Observer Spatial Degrees of Freedom Mimic A Proper Mix- ture}

No restriction has been placed on the initial probability amplitude $\psi^{\mathcal{Z}}(\vec{\zeta})$ for the translational degrees of freedom of the observer. How it comes about that there is nevertheless no conflict with the observed spatial localization of macroscopic objects such as measuring devices will be examined in the following section. Here we address a potential objection based on the form of the results (80), (82). Namely, these expressions for the probability of measurement outcomes explicitly involve the measuring-device-position amplitude $\psi^{\mathcal{Z}}(\vec{\zeta})$. Now, typically, when computing the respective probabilities of the possible outcomes of measurement of a quantum system performed using a macroscopic measuring device, one makes use of the state vector of the measured system; but one does not have to take into account any sort of wavefunction for the measuring device itself.

This apparent discrepancy is resolved by noting that the results (80), (82) of the Heisenberg-picture formalism are equal to those obtained if one proceeds in the usual manner - i.e., ignoring spatial degrees of freedom of the measuring apparatus - and computes the probabilities of measurement outcomes for a measured system initially in a mixed state. Since, from (49) and (154), $f(\vec{\zeta}, \vec{\xi})$ and $\widetilde{f}(\vec{\zeta}, \vec{\xi})$ are translation invariant,

$$
f(\vec{\zeta}, \vec{\xi})=f(\vec{\zeta}-\vec{q}, \vec{\xi}-\vec{q}) \quad \forall \vec{q}
$$




$$
\widetilde{f}(\vec{\zeta}, \vec{\xi})=\widetilde{f}(\vec{\zeta}-\vec{q}, \vec{\xi}-\vec{q}) \quad \forall \vec{q},
$$

(80), (82) can be written as

$$
\begin{gathered}
W_{b, 0}=\sum_{i=1}^{M} \int d^{3} \vec{\zeta} d^{3} \vec{\xi}\left|\psi^{\mathcal{Z}}(\vec{\zeta})\right|^{2} \widetilde{f}(\overrightarrow{0}, \vec{\xi})\left|\psi_{i}^{\mathcal{X} \mathcal{S}}(\vec{\xi}+\vec{\zeta})\right|^{2}, \\
W_{b, i}=\int d^{3} \vec{\zeta} d^{3} \vec{\xi}\left|\psi^{\mathcal{Z}}(\vec{\zeta})\right|^{2} f(\overrightarrow{0}, \vec{\xi})\left|\psi_{i}^{\mathcal{X S}}(\vec{\xi}+\vec{\zeta})\right|^{2}, \quad i=1, \ldots, M,
\end{gathered}
$$

where $\overrightarrow{0}=(0,0,0)$. Expressions (86) and (87) are precisely the values obtained in the usual formalism - i..e, disregarding the translational degrees of freedom $\mathcal{Z}$ of the measuring device - for the probabilities of obtaining the given outcomes upon measuring a classical statistical mixture (proper mixture - see Sec. 7.2 of Ref. 18) of quantum systems, with the (continuous) elements of the mixture indexed by $\vec{\zeta}$ and having the classical probability density $p(\vec{\zeta})=\left|\psi^{\mathcal{Z}}(\vec{\zeta})\right|^{2}$.

To see this, we redo the analysis that led to (86), (87), leaving out the observer translational degrees of freedom $\mathcal{Z}$ and taking the initial state to be a proper mixture. The state space is spanned by tensor products of the basis vectors (11), (2), and (29) (but not (301)). Define the $\mathcal{X}$-space projection operator

$$
\widehat{P}_{g}^{\mathcal{X}}=\int d^{3} \vec{\xi} g(\vec{\xi}) \widehat{P}_{\vec{\xi}}^{\mathcal{X}},
$$

where $g(\vec{\xi})$ is a c-number function satisfying

$$
(g(\vec{\xi}))^{2}=g(\vec{\xi}),
$$

so

$$
g(\vec{\xi})=0 \text { or } 1 \quad \forall \vec{\xi} .
$$

If we choose

$$
g(\vec{\xi})=\theta(a-|\vec{\xi}|), \quad a>0,
$$

i.e.,

$$
g(\vec{\xi})=f(\overrightarrow{0}, \vec{\xi}) .
$$

then $\widehat{P}_{g}^{\mathcal{X}}$ is the projector into $\mathcal{X}$-states within distance $a$ of the origin,

$$
\begin{aligned}
\widehat{P}_{g}^{\mathcal{X}}|\mathcal{X} ; \vec{\xi}\rangle & =|\mathcal{X} ; \vec{\xi}\rangle, & & |\vec{\xi}|<a, \\
& =0, & & |\vec{\xi}|>a .
\end{aligned}
$$

To model a finite-range measurement, we take the Hamiltonian ${ }^{3}$ to be

$$
\widehat{H}_{F}^{\prime}=\widehat{H}_{I} \otimes \widehat{P}_{g}^{\mathcal{X}},
$$

where $\widehat{H}_{I}$ is as given in (15). The time-evolution operator from the initial time $t_{i n}$ to time $t$,

$$
\widehat{U}_{F}^{\prime}=\exp \left(-i \widehat{H}_{F}^{\prime}\left(t-t_{i n}\right)\right),
$$

\footnotetext{
${ }^{3}$ Subsequent to the completion of this work I became aware of Sec. III of Ref. 23, where essentially the same Hamiltonian as in (94) appears.
} 
has the form

$$
\widehat{U}_{F}^{\prime}=\widehat{P}_{\vec{g}}^{\mathcal{X}}+\sum_{i=1}^{M} \widehat{u}_{i}^{\mathcal{O}} \otimes \widehat{P}_{i}^{\mathcal{S}} \otimes \widehat{P}_{g}^{\mathcal{X}}
$$

Here

$$
\widetilde{g}(\vec{\xi})=1-g(\vec{\xi})
$$

i.e.,

$$
\widetilde{g}(\vec{\xi})=\widetilde{f}(\overrightarrow{0}, \vec{\zeta})
$$

SO

$$
\widehat{P}_{\widetilde{g}}=\int d^{3} \vec{\xi} \widetilde{g}(\vec{\xi}) \widehat{P}_{\vec{\xi}}^{\mathcal{X}}
$$

$\widehat{P}_{\widetilde{g}}^{\mathcal{X}}$ is the projector into the subspace of states in $\mathcal{X}$ which are farther from the origin than $a$ :

$$
\begin{aligned}
\widehat{P}_{\vec{g}}^{\mathcal{X}}|\mathcal{X} ; \vec{\xi}\rangle & =|\mathcal{X} ; \vec{\xi}\rangle, & & |\vec{\xi}|>a, \\
& =0, & & |\vec{\xi}|<a .
\end{aligned}
$$

satisfying, from (39), (45), (88), (89) and (97)-(99),

$$
\begin{gathered}
\left(\widehat{P}_{\tilde{g}}^{\mathcal{X}}\right)^{2}=\widehat{P}_{\widetilde{g}}^{\mathcal{X}}, \\
\widehat{P}_{\tilde{g}}^{\mathcal{X}}=1-\widehat{P}_{g}^{\mathcal{X}}, \\
\widehat{P}_{\tilde{g}}^{\mathcal{X}} \widehat{P}_{g}^{\mathcal{X}}=\widehat{P}_{g}^{\mathcal{X}} \widehat{P}_{\widetilde{g}}^{\mathcal{X}}=0 .
\end{gathered}
$$

Using (17), (25), (65), (196), (101) and (103), the measurement-result operator $\hat{b}$ has, at time $t$, the value

$$
\widehat{b}^{\prime}(t)=\widehat{b}_{0} \otimes \widehat{P}_{\widetilde{g}}^{\mathcal{X}}+\sum_{i=1}^{M} \widehat{b}_{i} \otimes \widehat{P}_{i}^{\mathcal{S}} \otimes \widehat{P}_{g}^{\mathcal{X}} .
$$

( $\widehat{a}$ and $\widehat{\vec{x}}$ are unchanged by the measurement:

$$
\begin{aligned}
& \widehat{a}^{\prime}(t)=\widehat{U}_{F}^{\prime \dagger} \widehat{a} \widehat{U}_{F}^{\prime}=\widehat{a}, \\
& \left.\widehat{\vec{x}}^{\prime}(t)=\widehat{U}_{F}^{\prime \dagger} \widehat{\vec{x}} \widehat{U}_{F}^{\prime}=\widehat{\vec{x}} .\right)
\end{aligned}
$$

We take the initial (pre-measurement) state to be a proper mixture of the vectors

$$
\left|\psi_{1}^{\prime}\left(t_{i n}\right), \vec{\zeta}\right\rangle=\left|\mathcal{O} ; \beta_{0}\right\rangle \sum_{i=1}^{M} \int d^{3} \vec{\xi} \psi_{i}^{\mathcal{X}}(\vec{\xi}+\vec{\zeta})|\mathcal{X} ; \vec{\xi}\rangle\left|\mathcal{S} ; \alpha_{i}\right\rangle, \quad-\vec{\infty}<\vec{\zeta}<\vec{\infty}
$$

with classical probability density

$$
\begin{gathered}
p(\vec{\zeta}) \geq 0, \quad-\vec{\infty}<\vec{\zeta}<\vec{\infty}, \\
\int d^{3} \vec{\zeta} p(\vec{\zeta})=1 .
\end{gathered}
$$

Were the initial state one of the pure states $\left|\psi_{1}{ }^{\prime}\left(t_{i n}\right), \vec{\zeta}\right\rangle$ we would conclude from (178), (104), (107) and interpretive rule 1 that the degree of freedom $\mathcal{O}$, initially in a state of 
ignorance at time $t_{i n}$, has at the post-measurement time $t$ split into $M+1$ Everett copies which have respectively obtained measurement results $\beta_{i}, i=0, \ldots, M$. One can regard the formalism of a proper mixture as a convenient notation for calculating the results of experiments performed on ordinary classical ensembles of quantum systems each one of which is a pure state. ${ }^{4}$ We can then conclude that in each of the quantum systems in the ensemble, the degree of freedom $\mathcal{O}$ has split into Everett copies as described above. If, on the other hand, we wish to consider that a single quantum system can be described by a density operator $\widehat{\rho}\left(t_{i n}\right)$ which is not a pure state, i.e., one for which

$$
\left(\widehat{\rho}\left(t_{i n}\right)\right)^{2} \neq \widehat{\rho}\left(t_{i n}\right),
$$

then we must extend in a straightforward manner the initial-state requirements (9) and (26) to accommodate the case of general (not necessarily pure) states. The initial density operator must be of the form

$$
\widehat{\rho}\left(t_{i n}\right)=\widehat{\rho}_{\text {obs }}\left(t_{i n}\right) \otimes \widehat{\rho}_{\text {sys }}\left(t_{i n}\right)
$$

where $\hat{\rho}_{\text {obs }}\left(t_{i n}\right)$ and $\hat{\rho}_{\text {sys }}\left(t_{i n}\right)$ act only in the state spaces of the observer and system, respectively, and where

$$
\widehat{b}_{i} \widehat{\rho}_{\text {obs }}\left(t_{i n}\right)=\beta_{i} \widehat{\rho}_{\text {obs }}\left(t_{i n}\right), \quad i=0, \ldots, M \text {. }
$$

For the mixture under consideration here, the density operator is

$$
\hat{\rho}^{\prime}\left(t_{i n}\right)=\int d^{3} \vec{\zeta} p(\vec{\zeta})\left|\psi^{\prime}\left(t_{i n}\right), \vec{\zeta}\right\rangle\left\langle\psi^{\prime}\left(t_{i n}\right), \vec{\zeta}\right|
$$

or, using (107),

$$
\hat{\rho}^{\prime}\left(t_{i n}\right)=\hat{\rho}_{\text {obs }}^{\prime}\left(t_{i n}\right) \otimes \hat{\rho}_{s y s}^{\prime}\left(t_{i n}\right)
$$

where

$$
\widehat{\rho}_{o b s}^{\prime}\left(t_{i n}\right)=\left|\mathcal{O} ; \beta_{0}\right\rangle\left\langle\mathcal{O} ; \beta_{0}\right|
$$

and

$$
\hat{\rho}_{\text {sys }}^{\prime}\left(t_{i n}\right)=\int d^{3} \vec{\zeta} p(\vec{\zeta}) \sum_{i, i=1}^{M} \int d^{3} \vec{\xi} d^{3} \vec{\xi}^{\prime} \psi_{i}^{\mathcal{X} \mathcal{S}^{*}}(\vec{\xi}+\vec{\zeta}) \psi_{j}^{\mathcal{X} \mathcal{S}}\left(\overrightarrow{\xi^{\prime}}+\vec{\zeta}\right)|\mathcal{X} ; \vec{\xi}\rangle\left|\mathcal{S} ; \alpha_{i}\right\rangle\left\langle\mathcal{X} ; \overrightarrow{\xi^{\prime}}\right|\left\langle\mathcal{S} ; \alpha_{j}\right|
$$

Using (26) and (115),

$$
\widehat{b}_{i} \widehat{\rho}_{o b s}^{\prime}\left(t_{i n}\right)=\beta_{i} \widehat{\rho}_{o b s}^{\prime}\left(t_{i n}\right), \quad i=0, \ldots, M
$$

Whichever way we choose to think about proper mixtures, the probabilities for the respective measurement outcomes are given by the weights

$$
W_{b, 0}^{\prime}=\int d^{3} \vec{\zeta} p(\vec{\zeta}) W_{b, 0, \vec{\zeta}}^{\prime}
$$

\footnotetext{
${ }^{4}$ This is not to say that any mixture can be regarded in a unique manner an ensemble of pure states; that statement would be false. See, e.g., Sec. 2.3 of Ref. 24.
} 
for "no measurement made," and

$$
W_{b, i}^{\prime}=\int d^{3} \vec{\zeta} p(\vec{\zeta}) W_{b, i, \vec{\zeta}}^{\prime}, \quad i=1, \ldots, M
$$

for "outcome $\beta_{i}$ measured," $i=1, \ldots, M$, where

$$
\begin{gathered}
W_{b, 0, \vec{\zeta}}^{\prime}=\left\langle\psi^{\prime}\left(t_{i n}\right), \vec{\zeta}\left|\widehat{P}_{\vec{g}}^{\mathcal{X}}\right| \psi^{\prime}\left(t_{i n}\right), \vec{\zeta}\right\rangle \\
W_{b, i, \vec{\zeta}}^{\prime}=\left\langle\psi^{\prime}\left(t_{i n}\right), \vec{\zeta}\left|\widehat{P}_{i}^{\mathcal{S}} \otimes \widehat{P}_{g}^{\mathcal{X}}\right| \psi^{\prime}\left(t_{i n}\right), \vec{\zeta}\right\rangle, \quad i=1, \ldots, M .
\end{gathered}
$$

Using (7), (16), (188), (199) and (107), we find

$$
\begin{gathered}
W_{b, 0}^{\prime}=\sum_{i=1}^{M} \int d^{3} \vec{\zeta} d^{3} \vec{\xi} p(\zeta) \widetilde{g}(\xi)\left|\psi_{i}^{\mathcal{X} \mathcal{S}}(\vec{\xi}+\vec{\zeta})\right|^{2} \\
W_{b, i}^{\prime}=\int d^{3} \vec{\zeta} d^{3} \vec{\xi} p(\zeta) g(\vec{\xi})\left|\psi_{i}^{\mathcal{X} \mathcal{S}}(\vec{\xi}+\vec{\zeta})\right|, \quad i=1, \ldots, M .
\end{gathered}
$$

Using (92) and (98), we see that these probabilities are identical to the corresponding probabilities (86), (87), with the identification

$$
p(\vec{\zeta})=\left|\psi^{\mathcal{Z}}(\vec{\zeta})\right|^{2}
$$

We conclude that, when a measuring device $\mathcal{O}$ performs a measurement on a system $\mathcal{S}$ by means of the finite-range interaction (51), no ambiguity is introduced by taking into account the translational degrees of freedom $\mathcal{Z}$ associated with $\mathcal{O}$, either in the possible outcomes or in their respective probabilities. This is true regardless of the manner in which the initial-time wave function depends on the $\mathcal{Z}$ degrees of freedom. The presence of this $\mathcal{Z}$-dependence affects the measurement probabilities in the same way that an initial mixed state for the measured system would in the absence of the $\mathcal{Z}$ degrees of freedom.

\section{Multiple Observers and Spatial Localization}

We have seen in Sec. 3 above that inclusion of spatial degrees of freedom does not prevent unambiguous determination of outcomes or their associated probabilities in the Everett interpretation. However, in addition to obtaining definite results for measurements, with corresponding probabilities consistent with those predicted by quantum mechanics, it is a property of measuring devices that they can be considered to have well-defined spatial locations. In this section we present a scenario involving measurements by multiple observers illustrating that this property of localization is present in the Heisenberg-picture Everett formalism, at least when the interactions responsible for the measurements are localized along the lines of Sec. 3 .

The measurement scenario is of the following form: Two observers $\mathcal{O}^{(1)}$ and $\mathcal{O}^{(2)}$ each perform localized measurements on a system $\mathcal{S}$. By virtue of the fact that these observers are localized in space, we expect the results of the paired measurements to be correlated in certain ways. To see if this is the case, a third observer $\mathcal{G}$ performs measurements on 
both $\mathcal{O}^{(1)}$ and $\mathcal{O}^{(2)}$. (In Everett quantum mechanics, we must always consider an explicit measurement of the results of measurements made by different observers if we wish to speak of correlations between those results. In the absence of such an additional measurement, we can only say, e.g., that $\mathcal{O}^{(1)}$ and $\mathcal{O}^{(2)}$ have each split into their respective Everett copies, but cannot talk about correlations between the copies.)

Specifically, the observers $\mathcal{O}^{(p)}, p=1,2$ wish to examine whether they are in agreement as to the spatial locations of objects around them. They each make use of measuring devices of the type described in Sec. 3], which determine the state of system $\mathcal{S}$ provided $\mathcal{S}$ is located within a distance $a^{(p)}$ of the origin of $\mathcal{O}^{(p)}$ 's coordinate system. The two observers first have to arrange to use a common coordinate system so that they can meaningfully compare their results. They do so by mounting both measuring devices on a common platform, so as to maintain a fixed displacement between the devices. (For simplicity we will assume that the orientation of the platform is fixed.) Let the measuring devices be adjusted so that $\mathcal{O}^{(p)}$ can determine the state of $\mathcal{S}$ if $\mathcal{S}$ is within distance $a^{(p)}$ of the location $\vec{d}^{(p)}$, where $\vec{d}^{(p)}$ is measured from the common origin of coordinates fixed in the platform. After the $\mathcal{O}^{(p)}$ 's have performed their measurements, an observer $\mathcal{G}$ measures their respective states of awareness. We take the interaction by means of which $\mathcal{G}$ measures the $\mathcal{O}^{(p)}$ 's to be such that, at the conclusion of the measurement process, $\mathcal{G}$ will with certainty know the state of the $\mathcal{O}^{(p)}$ 's. (E.g., the $\mathcal{G}$ measuring apparatus might be mounted on the same platform as $\mathcal{O}^{(p)}$ 's in such a way that both of the $\mathcal{O}^{(p)}$ 's are within range.)

In other words, all three observers are here regarded as a single "bound structure." The spatial location of this structure, in addition to that of the spatial location of the to-be-measured system $\mathcal{S}$, is a quantum observable and, depending on the nature of the quantum state vector, is in general "smeared out." Is this state of affairs consistent with our usual notions of localization in space? In the present scenario, these notions lead us to expect the following results to be true:

Case 1. If $\left|\vec{d}^{(1)}-\vec{d}^{(2)}\right|>a^{(1)}+a^{(2)}$-i.e., if the regions in which the two measuring devices can interact with $\mathcal{S}$ have no overlap - then $\mathcal{G}$ will never find that both $\mathcal{O}^{(1)}$ and $\mathcal{O}^{(2)}$ have determined the state of $\mathcal{S}$.

Case 2. If, on the other hand, $\vec{d}^{(1)}=\vec{d}^{(2)}$ and $a^{(1)}=a^{(2)}$, so the two interaction regions coincide, then $\mathcal{G}$ will never find that only one of the $\mathcal{O}^{(p)}$ 's has determined the state of $\mathcal{S}$. Rather, $\mathcal{G}$ will always find either that neither has, or that both have, with the results obtained in the latter case by $\mathcal{O}^{(1)}$ and $\mathcal{O}^{(2)}$ always being in agreement.

\subsection{State Spaces}

The state space of the observed system is the same as that employed in Sec. 3, its location corresponding to the operator $\widehat{\vec{x}}$ and its internal state to the operator $\widehat{a}$. The spatial location of the observers is described by a single spatial coordinate which we will take to be the $\widehat{\vec{z}}$ of Sec. 3.1.1. In other words, the continuous degrees of freedom are the same as in Sec. 3 ,

The operators $\widehat{b}^{(p)}$ represent the states of awareness of the two observers who measure $\mathcal{S}:$

$$
\widehat{b}^{(p)}\left|\mathcal{O}^{(p)} ; \beta_{i}\right\rangle=\beta_{i}\left|\mathcal{O}^{(p)} ; \beta_{i}\right\rangle, \quad i=0, \ldots, M, \quad p=1,2,
$$


with the $\beta_{i}$ nondegenerate (see (6) ) and the $\left|\mathcal{O}^{(p)} ; \beta_{i}\right\rangle$ orthonormal,

$$
\left\langle\mathcal{O}^{(p)} ; \beta_{i} \mid \mathcal{O}^{(p)} ; \beta_{j}\right\rangle=\delta_{i j}, \quad i, j=O, \ldots, M, \quad p=1,2 .
$$

The state of awareness of $\mathcal{G}$, the observer who measures the respective states of awareness of $\mathcal{O}^{(1)}$ and $\mathcal{O}^{(2)}$ after they've measured $\mathcal{S}$, is given by the eigenvalues $\gamma_{I}$ of the operator $\widehat{g}$, with eigenvectors $\left|\mathcal{G} ; \gamma_{I}\right\rangle$,

$$
\widehat{g}\left|\mathcal{G} ; \gamma_{I}\right\rangle=\gamma_{I}\left|\mathcal{G} ; \gamma_{I}\right\rangle, \quad I=0,1, \ldots,(M+1)^{2} .
$$

$I=0$ indicates the state of ignorance, while the remaining $(M+1)^{2}$ possible values of $I$ correspond to $\mathcal{G}$ having determined that $\mathcal{O}^{(1)}$ has measured $\beta_{i}$ and that $\mathcal{O}^{(2)}$ has measured $\beta_{j}, i, j=0, \ldots, M$. That is, there is a mapping $I(i, j)$ from the pairs of states $\left|\mathcal{O}^{(1)} ; \beta_{i}\right\rangle\left|\mathcal{O}^{(2)} ; \beta_{j}\right\rangle$ which $\mathcal{G}$ observes to the $(M+1)^{2}$ non-ignorant states of awareness of $\mathcal{G}$.

\subsection{Measurement Interactions}

Define

$$
\begin{aligned}
f_{(p)}^{\prime \prime}(\vec{\zeta}, \vec{\xi}) & =\theta\left(a^{(p)}-\left|\vec{\xi}-\left(\vec{\zeta}+\vec{d}_{(p)}\right)\right|\right), \quad a^{(p)}>0, \quad p=1,2, \\
\tilde{f}_{(p)}^{\prime \prime}(\vec{\zeta}, \vec{\xi}) & =1-f_{(p)}^{\prime \prime}(\vec{\zeta}, \vec{\xi}), \quad p=1,2, \\
\widehat{P}_{f^{\prime \prime}(p)}^{\mathcal{Z}} & =\int d^{3} \vec{\zeta} d^{3} \vec{\xi} \widehat{P}_{\vec{\zeta}}^{\mathcal{Z}} \widehat{P}_{\vec{\xi}}^{\mathcal{X}} f_{(p)}^{\prime \prime}(\vec{\zeta}, \vec{\xi}), \quad p=1,2, \\
\widehat{P}_{\tilde{f}^{\prime \prime}(p)}^{\mathcal{Z}} & =\int d^{3} \vec{\zeta} d^{3} \vec{\xi} \widehat{P}_{\vec{\zeta}}^{\mathcal{Z}} \widehat{P}_{\vec{\xi}}^{\mathcal{X}} \widetilde{f}_{(p)}^{\prime \prime}(\vec{\zeta}, \vec{\xi})=1-\widehat{P}_{f^{\prime \prime}(p)}, \quad p=1,2 .
\end{aligned}
$$

The time evolution operators corresponding to the measurement of $\mathcal{S}$ by the $\mathcal{O}^{(p)}$ 's are then

$$
\widehat{U}_{F}^{(p) \prime \prime}=\widehat{P}_{f^{\prime \prime}(p)}^{\mathcal{Z} \mathcal{X}}+\sum_{i=1}^{M} \widehat{u}_{i}^{\mathcal{O}(p)} \otimes \widehat{P}_{i}^{\mathcal{S}} \otimes \widehat{P}_{f^{\prime \prime}(p)}^{\mathcal{Z} \mathcal{X}}, \quad p=1,2 .
$$

obtained from the Hamiltonians

$$
\widehat{H}_{F}^{(p) \prime \prime}=\sum_{i=1}^{M} \widehat{h}_{i}^{\mathcal{O}(p)} \otimes \widehat{P}_{i}^{\mathcal{S}} \otimes \widehat{P}_{f^{\prime \prime}(p)}^{\mathcal{Z X}}, \quad p=1,2,
$$

where

$$
\widehat{U}_{F}^{(p) \prime \prime}=\exp \left(-i \tau^{\prime \prime} \widehat{H}_{F}^{(p) \prime \prime}\right), \quad p=1,2 .
$$

$\widehat{u}_{i}^{\mathcal{O}(p)}$ is related to $\widehat{h}_{i}^{\mathcal{O}(p)}$ by

$$
\widehat{u}_{i}^{\mathcal{O}(p)}=\exp \left(-i \tau^{(p)} \widehat{h}_{i}^{\mathcal{O}(p)}\right), \quad i=1, \ldots, M, \quad p=1,2,
$$

with

$$
\widehat{h}_{i}^{\mathcal{O}(p)}=i \kappa^{(p)}\left(\left|\mathcal{O}^{(p)} ; \beta_{i}\right\rangle\left\langle\mathcal{O}^{(p)} ; \beta_{0}|-| \mathcal{O}^{(p)} ; \beta_{0}\right\rangle\left\langle\mathcal{O}^{(p)} ; \beta_{i}\right|\right) \quad p=1,2
$$

and

$$
\kappa^{(p)}=\frac{\pi}{2 \tau^{(p)}}, \quad p=1,2
$$


The time evolution operator corresponding to the measurement of the $\mathcal{O}^{(p)}$ 's by $\mathcal{G}$ is

$$
\widehat{U}_{F}^{\mathcal{G} \prime \prime}=\sum_{i, j=0}^{M} \widehat{u}_{i j}^{\mathcal{G}} \otimes \widehat{P}_{i}^{\mathcal{O}(1)} \otimes \widehat{P}_{j}^{\mathcal{O}(2)}
$$

related to the Hamiltonian

$$
\widehat{H}_{I}^{\mathcal{G}}=\sum_{i, j=0}^{M} \widehat{h}_{i j}^{\mathcal{G}} \otimes \widehat{P}_{i}^{\mathcal{O}(1)} \otimes \widehat{P}_{j}^{\mathcal{O}(2)}
$$

by

$$
\widehat{U}_{F}^{\mathcal{G}}{ }^{\prime \prime}=\exp \left(-i \tau^{\mathcal{G}} \widehat{H}_{I}^{\mathcal{G}}\right)
$$

where

$$
\begin{gathered}
\widehat{u}_{i j}^{\mathcal{G}}=\exp \left(-i \tau^{\mathcal{G}} \widehat{h}_{i j}^{\mathcal{G}}\right), \quad i, j=0, \ldots, M, \\
\widehat{h}_{i j}^{\mathcal{G}}=i \kappa^{\mathcal{G}}\left(\left|\mathcal{G} ; \gamma_{I(i, j)}\right\rangle\left\langle\mathcal{G} ; \gamma_{0}|-| \mathcal{G} ; \gamma_{0}\right\rangle\left\langle\mathcal{G} ; \gamma_{I(i, j)}\right|\right), \quad i, j=0, \ldots, M,
\end{gathered}
$$

and

$$
\kappa^{\mathcal{G}}=\frac{\pi}{2 \tau^{\mathcal{G}}}
$$

\subsection{Time Evolution of Operators}

The operator which generates time evolution from time $t_{i n}$ to time $t$ is

$$
\widehat{U}_{F 2}^{\prime \prime}=\widehat{U}_{F}^{\mathcal{G}}{ }^{\prime \prime} \widehat{U}_{F}^{(2) \prime \prime} \widehat{U}_{F}^{(1) \prime \prime}
$$

and the operator corresponding to the state of awareness of $\mathcal{G}$ at time $t$ is

$$
\begin{aligned}
\widehat{g}^{\prime \prime}(t) & =\widehat{U}_{F 2}^{\prime \prime \dagger} \widehat{g} \widehat{U}_{F 2}^{\prime \prime} \\
& =\sum_{i, j=0}^{M} \widehat{g}_{i j} \otimes \widehat{l}_{g, i j}^{\prime \prime}
\end{aligned}
$$

where

$$
\widehat{g}_{i j}=\widehat{u}_{i j}^{\mathcal{G} \dagger} \widehat{g} \widehat{u}_{i j}^{\mathcal{G}}
$$

and

$$
\begin{aligned}
\widehat{l}_{g, i j}^{\prime \prime}= & \widehat{P}_{i}^{\mathcal{O}(1)} \otimes \widehat{P}_{j}^{\mathcal{O}(2)} \otimes \widehat{P}_{\widetilde{f}^{\prime \prime}(1)}^{\mathcal{Z} \mathcal{X}} \widehat{P}_{\widetilde{f}^{\prime \prime}(2)}^{\mathcal{Z} \mathcal{X}} \\
& +\sum_{i^{\prime}=1}^{M}\left\{\widehat{u}_{i^{\prime}}^{\mathcal{O}(1) \dagger} \widehat{P}_{i}^{\mathcal{O}(1)} \widehat{u}_{i^{\prime}}^{\mathcal{O}(1)} \otimes \widehat{P}_{j}^{\mathcal{O}(2)} \otimes \widehat{P}_{f^{\prime \prime}(1)}^{\mathcal{Z} \mathcal{X}} \widehat{P}_{\widetilde{f}^{\prime \prime}(2)}^{\mathcal{Z X}}\right. \\
& +\widehat{P}_{i}^{\mathcal{O}(1)} \otimes \widehat{u}_{i^{\prime}}^{\mathcal{O}(2) \dagger} \widehat{P}_{j}^{\mathcal{O}(2)} \widehat{u}_{i^{\prime}}^{\mathcal{O}(2)} \otimes \widehat{P}_{\widetilde{f}^{\prime \prime}(1)}^{\mathcal{Z} \mathcal{P}} \widehat{P}_{f^{\prime \prime}(2)}^{\mathcal{Z} \mathcal{X}} \\
& \left.+\widehat{u}_{i^{\prime}}^{\mathcal{O}(1) \dagger} \widehat{P}_{i}^{\mathcal{O}(1)} \widehat{u}_{i^{\prime}}^{\mathcal{O}(1)} \otimes \widehat{u}_{i^{\prime}}^{\mathcal{O}(2) \dagger} \widehat{P}_{j}^{\mathcal{O}(2)} \widehat{u}_{i^{\prime}}^{\mathcal{O}(2)} \otimes \widehat{P}_{f^{\prime \prime}(1)}^{\mathcal{Z} \mathcal{X}} \widehat{P}_{f^{\prime \prime}(2)}^{\mathcal{Z} \mathcal{X}}\right\} \otimes \widehat{P}_{i^{\prime}}^{\mathcal{S}}
\end{aligned}
$$

satisfying

$$
\widehat{l}_{g, i j}^{\prime \prime} \widehat{l}_{g, i^{\prime} j^{\prime}}^{\prime \prime}=\widehat{l}_{g, i j}^{\prime \prime} \delta_{i i^{\prime}} \delta_{j j^{\prime}}
$$


and

$$
\sum_{i, j=0}^{M} \widehat{l}_{g, i j}^{\prime \prime}=1
$$

(The operators $\widehat{b}^{(p)}$ undergo Everett splitting, while $\widehat{a}, \widehat{\vec{x}}$ and $\widehat{\vec{z}}$ are unchanged:

$$
\begin{aligned}
\widehat{b}^{(p)}(t) & =\widehat{U}_{F}^{\prime \prime \dagger} \widehat{b}^{(p)} \widehat{U}_{F}^{\prime \prime}=\widehat{b}_{0}^{(p)} \otimes \widehat{P}_{\tilde{f}(p)}^{\mathcal{Z} \mathcal{X}}+\sum_{i=1}^{M} \widehat{b}_{i}^{(p)} \otimes \widehat{p}_{i}^{\mathcal{S}} \otimes \widehat{p}_{f(p)}^{\mathcal{Z} \mathcal{X}}, \quad p=1,2, \\
\widehat{a}(t) & =\widehat{U}_{F}^{\prime \prime \dagger} \widehat{a} \widehat{U}_{F}^{\prime \prime}=\widehat{a} \\
\widehat{\vec{x}}(t) & =\widehat{U}_{F}^{\prime \prime \dagger} \widehat{\vec{x}} \widehat{U}_{F}^{\prime \prime}=\widehat{\vec{x}} \\
\widehat{\vec{z}}(t) & \left.=\widehat{U}_{F}^{\prime \prime \dagger} \widehat{\vec{z}} \widehat{U}_{F}^{\prime \prime}=\widehat{\vec{z}} .\right)
\end{aligned}
$$

\subsection{Initial State}

The observables pertaining to the measured system should be uncorrelated with those pertaining to the measuring devices. In addition all three measuring devices should be in their respective states of ignorance. We therefore take the initial state to be

$$
\left|\psi_{2}^{\prime \prime}\left(t_{\text {in }}\right)\right\rangle=\left|\mathcal{G}, \mathcal{Z}, \mathcal{O}, t_{\text {in }}\right\rangle\left|\mathcal{X}, \mathcal{S}, t_{\text {in }}\right\rangle,
$$

where

$$
\left|\mathcal{G}, \mathcal{Z}, \mathcal{O}, t_{i n}\right\rangle=\int d^{3} \vec{\zeta} \psi^{\mathcal{Z}}(\vec{\zeta})|\mathcal{Z} ; \vec{\zeta}\rangle\left|\mathcal{G} ; \gamma_{0}\right\rangle\left|\mathcal{O}^{(1)} ; \beta_{0}\right\rangle\left|\mathcal{O}^{(2)} ; \beta_{0}\right\rangle
$$

and where $\left|\mathcal{X}, \mathcal{S}, t_{\text {in }}\right\rangle$ is as in eq. (174). Normalization of $\left|\psi_{2}^{\prime \prime}\left(t_{i n}\right)\right\rangle$,

$$
\left\langle\psi_{2}^{\prime \prime}\left(t_{i n}\right) \mid \psi_{2}^{\prime \prime}\left(t_{i n}\right)\right\rangle=1
$$

imposes the constraint

$$
\left(\int d^{3} \vec{\xi} \sum_{i=1}^{M}\left|\psi_{i}^{\mathcal{X} \mathcal{S}}(\vec{\xi})\right|^{2}\right)\left(\int d^{3} \vec{\zeta}\left|\psi^{\mathcal{Z}}(\vec{\zeta})\right|^{2}\right)=1 .
$$

\subsection{Probability}

The probability that $\mathcal{G}$ determines that the states of awareness of $\mathcal{O}^{(1)}$ and $\mathcal{O}^{(2)}$ are respectively $\beta_{i}$ and $\beta_{j}$ is

$$
W_{2 g, i j}^{\prime \prime}=\left\langle\psi_{2}^{\prime \prime}\left(t_{i n}\right)\left|\widehat{l}_{g, i j}^{\prime \prime}\right| \psi_{2}^{\prime \prime}\left(t_{i n}\right)\right\rangle,
$$

Using (30,42), (174), (128, 131), (149), (155) and (156) in (159), we find that

$$
\begin{aligned}
W_{2 g, i j}^{\prime \prime}= & \int d^{3} \vec{\zeta} d^{3} \vec{\xi}\left|\psi^{\mathcal{Z}}(\vec{\zeta})\right|^{2} \\
& \cdot\left[\delta_{i 0} \delta_{j 0} \widetilde{f}_{(1)}(\vec{\zeta}, \vec{\xi}) \tilde{f}_{(2)}^{\prime \prime}(\vec{\zeta}, \vec{\xi}) \sum_{k=1}^{M}\left|\psi_{k}^{\mathcal{X S}}(\vec{\xi})\right|^{2}\right. \\
& +\left(1-\delta_{i 0}\right) \delta_{j 0} f_{(1)}^{\prime \prime}(\vec{\zeta}, \vec{\xi}) \tilde{f}_{(2)}^{\prime \prime}(\vec{\zeta}, \vec{\xi})\left|\psi_{i}^{\mathcal{X}}(\vec{\xi})\right|^{2} \\
& +\delta_{i 0}\left(1-\delta_{j 0}\right) \widetilde{f}_{(1)}^{\prime \prime}(\vec{\zeta}, \vec{\xi}) f_{(2)}^{\prime \prime}\left(\vec{\zeta}^{\prime}, \vec{\xi}\right)\left|\psi_{j}^{\mathcal{X}}(\vec{\xi})\right|^{2} \\
& \left.+\delta_{i j}\left(1-\delta_{i 0}\right) f_{(1)}^{\prime \prime}(\vec{\zeta}, \vec{\xi}) f_{(2)}^{\prime \prime}(\vec{\zeta}, \vec{\xi})\left|\psi_{i}^{\mathcal{X}}(\vec{\xi})\right|^{2}\right], \quad i, j=0, \ldots, M .
\end{aligned}
$$


Using (128), (129), (158) and (160) we verify that

$$
\sum_{i, j=0}^{M} W_{2 g, i j}^{\prime \prime}=1 .
$$

We now consider the two possibilities for the value of the $\vec{d}^{(p)}$ 's and the $a^{(p)}$ 's described above:

Case 1. Suppose that

$$
\left|\vec{d}^{(1)}-\vec{d}^{(2)}\right|>a^{(1)}+a^{(2)}
$$

Then

$$
f_{(1)}^{\prime \prime}(\vec{\zeta}, \vec{\xi}) f_{(2)}^{\prime \prime}(\vec{\zeta}, \vec{\xi})=0 \quad \forall \vec{\zeta}, \vec{\xi}
$$

To verify this, assume that, on the contrary,

$$
f_{(1)}^{\prime \prime}\left(\overrightarrow{\zeta^{\prime}}, \overrightarrow{\xi^{\prime}}\right) f_{(2)}^{\prime \prime}\left(\vec{\zeta}^{\prime}, \vec{\xi}^{\prime}\right) \neq 0
$$

for some $\overrightarrow{\zeta^{\prime}}, \overrightarrow{\xi^{\prime}}$, implying

$$
\begin{aligned}
f_{(1)}^{\prime \prime}\left(\vec{\zeta}^{\prime}, \vec{\xi}^{\prime}\right) & \neq 0, \\
f_{(2)}^{\prime \prime}\left(\vec{\zeta}^{\prime}, \vec{\xi}^{\prime}\right) & \neq 0 .
\end{aligned}
$$

Using the definition (128), these imply that

$$
\begin{aligned}
& \left|\vec{\xi}^{\prime}-\left(\vec{\zeta}^{\prime}+\vec{d}^{(1)}\right)\right|<a^{(1)}, \\
& \left|\vec{\xi}^{\prime}-\left(\vec{\zeta}^{\prime}+\vec{d}^{(2)}\right)\right|<a^{(2)},
\end{aligned}
$$

So

$$
\left|\vec{\xi}^{\prime}-\left(\vec{\zeta}^{\prime}+\vec{d}^{(1)}\right)\right|+\left|\vec{\xi}^{\prime}-\left(\vec{\zeta}^{\prime}+\vec{d}^{(2)}\right)\right|<a^{(1)}+a^{(2)} .
$$

It follows from the Schwartz inequality that, for any vectors $\vec{A}$ and $\vec{B}$,

$$
|\vec{A}-\vec{B}| \leq|\vec{A}|+|\vec{B}|
$$

(see, e.g., p. 35 of Ref. 25).Therefore (169) implies

$$
\left|\vec{d}^{(1)}-\vec{d}^{(2)}\right| \leq a^{(1)}+a^{(2)},
$$

contradicting the condition (162).

So, in this case - i.e., "large" separation, as given by (162) - the last term in the square brackets in (160) vanishes, implying $W_{2 g, i j}^{\prime \prime}=0$ unless at least one of the indices $i, j$ is zero. That is, there is zero probability that $\mathcal{O}^{(1)}$ and $\mathcal{O}^{(2)}$ both measure $\mathcal{S}$.

Case 2. If $\vec{d}^{(1)}=\vec{d}^{(2)}$ and $a^{(1)}=a^{(2)}$ then, from (128),

$$
f_{(1)}^{\prime \prime}(\vec{\zeta}, \vec{\xi})=f_{(2)}^{\prime \prime}(\vec{\zeta}, \vec{\xi}) \text {. }
$$

From (128) and (129)

$$
f_{(p)}^{\prime \prime}(\vec{\zeta}, \vec{\xi}) \tilde{f}_{(p)}^{\prime \prime}(\vec{\zeta}, \vec{\xi})=0, \quad p=1,2 .
$$

Eqs. (172) and (173) imply that the second and third terms in the square brackets in (160) vanish. So, in this case of both $\mathcal{O}^{(1)}$ and $\mathcal{O}^{(2)}$ measuring $\mathcal{S}$ in the same location, $W_{2 g, i j}^{\prime \prime}=0$ unless both of the indices $i, j$ are the same. $\mathcal{G}$ will always observe either that both $\mathcal{O}^{(1)}$ and $\mathcal{O}^{(2)}$ have failed to measure $\mathcal{S}$, or that both have measured it and obtained the same value. 


\section{Summary and Discussion}

When applied to the localized measurement interaction model developed in this paper, the rules for determining the possible results of measurements and their associated probabilities in the Heisenberg-picture Everett interpretation give unambiguous results in the presence of continuous translational degrees of freedom for both measured systems and observers just as they do in their absence. The continuous-spectrum translational degrees of freedom of the measuring device have the same effect on the measurement results as would a mixed state for the observed system.

Despite the presence of translational degrees of freedom for observers, the results obtained by multiple observers measuring another system and subsequently comparing their results will accord with what we think of when we say that a macroscopic system like a measuring device is "in one place at a time." E.g., if the observation regions of the observers $\mathcal{O}^{(1)}$ and $\mathcal{O}^{(1)}$ in Sec. 4 are well-separated, they will never both measure $\mathcal{S}$; if those regions coincide, they will always measure it, or fail to measure it, in coincidence, and with the same results when they do measure it.

To the extent that Stapp's claim that macroscopic objects, in particular measuring devices, are "smeared out over a continuum of locations" in collapse-free Everett quantum mechanics is taken to be a statement of underlying ontology, we have not refuted it here. Rather, we have argued that such smearing will not be perceived. The examples of Secs. 3 and 4 indicate that, to the extent that observers operationally determine their positions in space by measuring the positions of objects using localized measurement interactions and then comparing their results, each Everett copy of an observer determining its location in such manner will obtain an unambiguous result, with a precision dictated by the limitations the apparatus employed. Thus, both internal and spatial properties of macroscopic objects are definite at what Schlosshauer (Sec. II.B.3 of Ref. 13) terms the "observational level." This is sufficient to put the theory in accord with experience; see Sec. II.B.3 of Ref. 13 and Ref. $26 . .^{5}$

The analysis presented in this paper is in the context of a specific finite-range interaction model. It is to be expected that the conclusions obtained through this analysis, particularly regarding localization of measuring devices, will carry over to more complicated and realistic measurement models, since these will also be finite-range. E.g., the best models we currently have of fundamental interactions, quantum field theories, ${ }^{6}$ involve basic constituents (operator-valued fields) which interact only at the same space-time point (see, e.g., Refs. 28 and 29).

Joos ${ }^{(30)}$ has previously pointed out the importance of variables with discrete spectra for the Everett interpretation, and Zurek, in a private communication reported by Schlosshauer, argues that the core basis problem vanishes at the observational level due to the approximately-discrete nature of the states of neurons in the human brain: "...It is ultimately only in the brain where the perception of denumerability and mutual exclusiveness of events must be accounted for...when neurons are more appropriately modeled as two-state systems, the issue raised by Stapp disappears..." (Sec. IV.C.1 of Ref. 13).

\footnotetext{
${ }^{5}$ Re: probability as an observational-level phenomenon, see Refs. 31-33.

${ }^{6}$ The standard model quantum field theory is believed to correctly describe particles and their interactions down to a length scale of $10^{-18} \mathrm{~m} . \frac{(27)}{27}$
} 
As I have argued in this paper, discrete states for observers are indeed a necessary ingredient for the resolution of the core basis problem. However, what is equally important is taking an operational approach to the properties of objects, including measuring devices. To obtain information about, say, the location of a measuring device, one must perform a measurement to determine it, either explictly or (as in Sec. 4) implicitly. Without such an approach it is unclear, e.g., how to handle the translational degrees of freedom of the measuring device while it is being used to measure the property $\mathcal{S}$ (see Sec. 3). The use of the Heisenberg-picture Everett formalism makes this operational approach essentially automatic, since the very definition of when something has a property (interpretational rule 1) is couched in terms of the time evolution, as a result of measurement interaction, of a degree of freedom of a measuring device.

Note that, since we are not employing the Copenhagen interpretation, there are no classical objects, with c-number locations in space, present in the formalism with respect to which to measure the position of quantum objects in any absolute sense. The only position information which can be obtained is relative information, as in Sec. 4. " $\mathcal{O}^{(2)}$ is (or is not) at the same location as $\mathcal{O}^{(1)}$."

(The approximately two-valued voltage across a neuron membrane is certainly sufficient to serve as an observer-type degree of freedom $\mathcal{O}$, but it seems unlikely that it is necessary to proceed so far up the ladder of complexity before encountering a suitable operator. Degrees of freedom in inorganic matter would seem to be adequate, e.g. those related to the excitation of vibrational states of a rock upon absorption of a photon. In the nervous system, the change in the geometry of a rhodopsin molecule upon absorption of light (see, e.g., Ch. 26 of Ref. 34) might be another example. ${ }^{7}$ Disturbance of the measured system upon measurement, as when a photon is absorbed, is another, different, departure from the ideal-measurement model, but a rather straightforward one (see, e.g., Sec. 9.2 of Ref. 24) which does not modify interpretational issues.)

Several open issues remain. As noted above (Sec. 3.1.5), the derivation of Born-rule probability $^{8}$ from relative frequency in the infinite-ensemble limit given in Ref. 16 for systems with discrete degrees of freedom has not to date been extended to system with continuous degrees of freedom such as those in the present paper. The analysis presented

\footnotetext{
${ }^{7}$ Rhodopsin, as the site of interaction between external electromagnetic fields and the human visual system, has the been the subject of a variety of proposals, both theoretical and experimental, regarding quantum measurement. ${ }^{(35-39)}$

${ }^{8}$ Recently van Esch ${ }^{40}$ has claimed that the Born rule is not derivable from the nonprobabilistic elements of the Everett formalism but must rather be an independent postulate. He defines, within the usual nonprobabilistic structure of quantum mechanics with discrete degrees of freedom, a rule which associates real numbers between zero and unity with Schrödinger-picture Everett branches. He refers to this rule as an "alternative probability rule," and then argues that since this probability rule, which differs from the Born probability rule, can be defined in the context of the nonprobabilistic elements of Everett quantum theory just as well as the Born rule, the Born rule cannot be derivable from the nonprobabilistic elements but must instead be an independent postulate. Van Esch retains in his alternative quantum theory properties equivalent to interpretive rules 1 and 2. Therefore, the results of Ref. 16 show that, in his alternative quantum theory, the probability rule would disagree with the results obtained by a relative-frequencymeasuring device in the infinite-ensemble limit (at least in the physically-interesting case that measuring devices are of finite size and employ finite amounts of energy in their operation). It is questionable whether such a rule, bearing no relation to long-term relative frequency, should be termed a "probability" rule at all.
} 
in this paper should also be extended to include rotational as well as translational degrees of freedom. The finite-range interaction Hamiltonian for, say, a Stern-Gerlach type device measuring the spin of a spin $1 / 2$ particle would differ somewhat from the form (51) the projection operators (16) would be replaced by projection operators dependent on the orientation of the measuring device, since which components of spin are measured depends on this orientation.

The nondegenerate eigenvalues of the $\mathcal{O}$ degree of freedom in any measurement model of the type presented here should correspond to "facts" - e.g., "current is flowing in the semiconductor junction," "light-emitting diode is on," "the voltage across this portion of the cell membrane has a certain value" - which are true or false independent of location, orientation or other degrees of freedom of the measuring apparatus. A genuinely internal isospin-like degree of freedom would of course fit the bill, but more relevant for modeling most measurement-like situations are systems in which the $\mathcal{O}$ degree of freedom is a function of coordinates describing the positions and/or motions of constituent pieces of the measuring apparatus relative to one another. So, the construction of simple but explicit models in which the $\mathcal{O}$ degree of freedom is constructed in this manner is essential to relate the abstract model presented above to such more realistic measurement devices, and to determine whether these devices too can be described without basis problems in an Everett framework.

Finally, having seen that well-defined isolated measurements can exist in a collapsefree quantum-mechanical model, we are still left with the question of why only certain types of measuring devices, and not others, are essentially always encountered in art and in nature. Our instruments and our brains can detect and distinguish between live cats and dead cats; but rare indeed are "Schrödinger-cat sensors" which detect superpositions of macroscopically-distinct states of observed systems. ${ }^{9}$ The answer to this question is nowadays thought to be decoherence: Due to interaction with the environment, successive measurements made by such detectors would in all but highly-controlled conditions give uncorrelated results, and so would have no predictive value. Such detectors would therefore not be built, nor would observers capable of making such measurements have evolved (Ref. 5; see also Sec. III.E.1 of Ref. 13 and references therein). There is no reason to think that decoherence cannot continue to play this role ${ }^{10}$ in models of the type presented in this paper, although of course this issue ought to be examined in detail. But, at least in the model presented above, decoherence is not required in order for the results of Everettquantum-mechanical measurements involving measured systems and measuring devices with discrete and translational degrees of freedom to be unambiguous and compatible with localization of the measuring devices.

\footnotetext{
${ }^{9}$ Rare, but not nonexistent! 41

${ }^{10}$ In addition, decoherence suppresses interference between possible trajectories of macroscopic objects. Interference is a phenomenon which involves properties of physical systems not just at individual times but at sequences of times (histories) (see Sec. II of Ref. 42). Quantitative analysis of interference suppression requires consideration of the free dynamics of the system between measurement interactions (Ref. 43; see also Ref. 44 and references therein) as well as the during-measurement interactions which have been considered here. Recently, suppression of interference through environmental decoherence has been demonstrated experimentally. ${ }^{(45-47)}$
} 


\section{Acknowledgments}

I would like to thank Jian-Bin Mao, Rainer Plaga, Allen J. Tino and two anonymous referees for very helpful discussions and comments.

\section{Appendix: A Generalized Operator Expansion Unique- ness Theorem}

In this Appendix we extend the operator expansion uniqueness theorem of Sec. 4.2.1 of Ref. 10 to systems with continuous degrees of freedom such as translation, in order to show that the form for $\widehat{b}(t)$ in (64) is unique.

Operator expansion uniqueness theorem for systems with continuous degrees of freedom: Let $\widehat{b}(t)$ be an operator which acts in the product space $\mathcal{O} \otimes \mathcal{V}$ and can be expanded as

$$
\widehat{b}(t)=\sum_{i=0}^{M} \widehat{b}_{i}^{\prime} \otimes \widehat{Q}_{i}^{\prime} .
$$

Each Hermitian operator $\widehat{b}_{i}^{\prime}, i=0, \ldots, M$ acts nontrivially only in the $(M+1)$-dimensional state space $\mathcal{O}$, and satisfies

$$
\begin{gathered}
\widehat{b}_{i}^{\prime}|\mathcal{O}: 0\rangle=\beta_{i}^{\prime}|\mathcal{O}: 0\rangle, \quad i=0, \ldots, M, \\
\beta_{i}^{\prime}=\beta_{j}^{\prime} \Rightarrow i=j, \quad i, j=0, \ldots, M,
\end{gathered}
$$

for some vector $|\mathcal{O}: 0\rangle$ in $\mathcal{O}$. Each Hermitian operator $\widehat{Q}_{i}^{\prime}, i=0, \ldots, M$, acts nontrivially only in the state space $\mathcal{V}$ which is spanned by basis vectors $|\mathcal{V}: \vec{l}, \vec{\nu}\rangle$ carrying both discrete and continuous indices:

$$
\begin{gathered}
\left\langle\mathcal{V}: \vec{l}, \vec{\nu} \mid \mathcal{V}: \vec{l}^{\prime}, \vec{\nu}^{\prime}\right\rangle=\delta_{\vec{l} \vec{l}^{\prime}} \delta\left(\vec{\nu}-\vec{\nu}^{\prime}\right), \\
\sum_{\vec{l}} \int d \vec{\nu}|\mathcal{V}: \vec{l}, \vec{\nu}\rangle\langle\mathcal{V}: \vec{l}, \vec{\nu}|=1
\end{gathered}
$$

The $\widehat{Q}_{i}^{\prime}$ 's form a complete set of nontrivial orthogonal projection operators in $\mathcal{V}$ :

$$
\begin{gathered}
\widehat{Q}_{i}^{\prime} \widehat{Q}_{j}^{\prime}=\delta_{i j} \widehat{Q}_{i}^{\prime}, \quad i=0, \ldots, M, \\
\sum_{i=0}^{M} \widehat{Q}_{i}^{\prime}=1, \\
\widehat{Q}_{i}^{\prime} \neq 0, \quad i=0, \ldots, M .
\end{gathered}
$$

Suppose that there exists another expansion for $\widehat{b}(t)$,

$$
\widehat{b}(t)=\sum_{i=0}^{M} \widehat{b}_{i}^{\prime \prime} \otimes \widehat{Q}_{i}^{\prime \prime},
$$

where the $\widehat{b}_{i}^{\prime \prime}$ 's are Hermitian operators acting in $\mathcal{O}$ and satisfying

$$
\widehat{b}_{i}^{\prime \prime}|\mathcal{O}: 0\rangle=\beta_{i}^{\prime \prime}|\mathcal{O}: 0\rangle, \quad i=0, \ldots, M
$$




$$
\beta_{i}^{\prime \prime}=\beta_{j}^{\prime \prime} \Rightarrow i=j, \quad i, j=0, \ldots, M,
$$

for the same vector $|\mathcal{O}: 0\rangle$ in $\mathcal{O}$, and where the $\widehat{Q}_{i}^{\prime \prime \prime}$ s are Hermitian operators acting in $\mathcal{V}$ satisfying

$$
\begin{gathered}
\widehat{Q}_{i}^{\prime \prime} \widehat{Q}_{j}^{\prime \prime}=\delta_{i j} \widehat{Q}_{i}^{\prime \prime}, \quad i=0, \ldots, M, \\
\sum_{i=0}^{M} \widehat{Q}_{i}^{\prime \prime}=1, \\
\widehat{Q}_{i}^{\prime \prime} \neq 0, \quad i=0, \ldots, M .
\end{gathered}
$$

Then the $\widehat{b}_{i}^{\prime \prime \prime}$ s and $\widehat{Q}_{i}^{\prime \prime \prime}$ s are identical to the $\widehat{b}_{i}^{\prime}$ 's and $\widehat{Q}_{i}^{\prime}$ 's up to renumbering:

$$
\begin{gathered}
\widehat{b}_{i}^{\prime \prime}=\widehat{b}_{\pi(i)}^{\prime}, \quad i=0, \ldots, M, \\
\widehat{Q}_{i}^{\prime \prime}=\widehat{Q}_{\pi(i)}^{\prime}, \quad i=0, \ldots, M,
\end{gathered}
$$

where $\pi(i)$ is some permutation of $i=0, \ldots, M$.

Proof: Since the $\widehat{Q}_{i}^{\prime}$ 's are Hermitian and commute (eq. (179)) they have a common set of eigenvectors $\left|\mathcal{V}^{\prime}: \vec{l}, \vec{\nu}\right\rangle$ which span $\mathcal{V}$ and which can be chosen to be orthonormal:

$$
\begin{gathered}
\widehat{Q}_{i}^{\prime}\left|\mathcal{V}^{\prime}: \vec{l}, \vec{\nu}\right\rangle=\omega_{i}^{\prime}(\vec{l}, \vec{\nu})\left|\mathcal{V}^{\prime}: \vec{l}, \vec{\nu}\right\rangle, \quad i=0, \ldots, M, \\
\left\langle\mathcal{V}^{\prime}: \vec{l}, \vec{\nu} \mid \mathcal{V}^{\prime}: \overrightarrow{l^{\prime}}, \vec{\nu}^{\prime}\right\rangle=\delta_{\vec{l} \overrightarrow{l^{\prime}}} \delta\left(\vec{\nu}-\vec{\nu}^{\prime}\right) . \\
\sum_{\vec{l}} \int d \vec{\nu}\left|\mathcal{V}^{\prime}: \vec{l}, \vec{\nu}\right\rangle\left\langle\mathcal{V}^{\prime}: \vec{l}, \vec{\nu}\right|=1, \\
\widehat{Q}_{i}^{\prime}=\sum_{\vec{l}} \int d \vec{\nu}\left|\mathcal{V}^{\prime}: \vec{l}, \vec{\nu}\right\rangle \omega_{i}^{\prime}(\vec{l}, \vec{\nu})\left\langle\mathcal{V}^{\prime}: \vec{l}, \vec{\nu}\right|,
\end{gathered}
$$

Since the $\omega_{i}^{\prime}(\vec{l}, \vec{\nu})^{\prime}$ 's are eigenvalues of projection operators,

$$
\omega_{i}^{\prime}(\vec{l}, \vec{\nu})=0 \text { or } 1
$$

Lemma 1: For fixed $\vec{l}, \vec{\nu}, \omega_{i}^{\prime}(\vec{l}, \vec{\nu})=1$ for exactly one value of $i ; \omega_{i}^{\prime}(\vec{l}, \vec{\nu})=0$ for all other values of $i$.

Proof: From (180),

$$
\sum_{i=0}^{M} \widehat{Q}_{i}^{\prime}\left|\mathcal{V}^{\prime}: \vec{l}, \vec{\nu}\right\rangle=\left|\mathcal{V}^{\prime}: \vec{l}, \vec{\nu}\right\rangle,
$$

so, using (190)

$$
\sum_{i=0}^{M} \omega_{i}^{\prime}(\vec{l}, \vec{\nu})\left|\mathcal{V}^{\prime}: \vec{l}, \vec{\nu}\right\rangle=\left|\mathcal{V}^{\prime}: \vec{l}, \vec{\nu}\right\rangle
$$

Using (177),

$$
\sum_{i=0}^{M} \omega_{i}^{\prime}(\vec{l}, \vec{\nu})=1 .
$$

This can only be consistent with (194) if, for any values of $\vec{l}$ and $\vec{\nu}, \omega_{i}^{\prime}(\vec{l}, \vec{\nu})$ is equal to unity for one value of $i$ and zero for all the others. 
Similarly, the $\widehat{Q}_{i}^{\prime \prime}$ 's have a common complete set of orthonormal eigenvectors:

$$
\begin{gathered}
\widehat{Q}_{i}^{\prime \prime}\left|\mathcal{V}^{\prime \prime}: \vec{l}, \vec{\nu}\right\rangle=\omega_{i}^{\prime \prime}(\vec{l}, \vec{\nu})\left|\mathcal{V}^{\prime \prime}: \vec{l}, \vec{\nu}\right\rangle, \quad i=0, \ldots, M, \\
\left\langle\mathcal{V}^{\prime \prime}: \vec{l}, \vec{\nu} \mid \mathcal{V}^{\prime \prime}: \overrightarrow{l^{\prime}}, \vec{\nu}^{\prime}\right\rangle=\delta_{\vec{l} l^{\prime}} \delta\left(\vec{\nu}-\vec{\nu}^{\prime}\right), \\
\sum_{\vec{l}} \int d \vec{\nu}\left|\mathcal{V}^{\prime \prime}: \vec{l}, \vec{\nu}\right\rangle\left\langle\mathcal{V}^{\prime \prime}: \vec{l}, \vec{\nu}\right|=1, \\
\widehat{Q}_{i}^{\prime \prime}=\sum_{\vec{l}} \int d \vec{\nu}\left|\mathcal{V}^{\prime \prime}: \vec{l}, \vec{\nu}\right\rangle \omega_{i}^{\prime \prime}(\vec{l}, \vec{\nu})\left\langle\mathcal{V}^{\prime \prime}: \vec{l}, \vec{\nu}\right|, \\
\omega_{i}^{\prime \prime}(\vec{l}, \vec{\nu})=0 \text { or } 1,
\end{gathered}
$$

with, for fixed $\vec{l}$ and $\vec{\nu}, \omega_{i}^{\prime \prime}(\vec{l}, \vec{\nu})$ equal to unity for one value of $i$ and equal to zero for all the other values of $i$.

Define functions which map from values of $\vec{l}$ and $\vec{\nu}$ to the subscript indices of the nonzero eigenvalues $\omega_{i}^{\prime}(\vec{l}, \vec{\nu}), \omega_{i}^{\prime \prime}(\vec{l}, \vec{\nu})$ :

$$
\begin{array}{lll}
I^{\prime}(\vec{l}, \vec{\nu})=i & \text { s.t. } \quad \omega_{i}^{\prime}(\vec{l}, \vec{\nu})=1 \\
I^{\prime \prime}(\vec{l}, \vec{\nu})=i & \text { s.t. } \quad \omega_{i}^{\prime \prime}(\vec{l}, \vec{\nu})=1 .
\end{array}
$$

In other words,

$$
\begin{aligned}
& \omega_{i}^{\prime}(\vec{l}, \vec{\nu})=\delta_{i, I^{\prime}(\vec{l}, \vec{\nu})} \\
& \omega_{i}^{\prime \prime}(\vec{l}, \vec{\nu})=\delta_{i, I^{\prime \prime}(\vec{l}, \vec{\nu})} .
\end{aligned}
$$

It will also be useful to define the sets

$$
\begin{aligned}
\mathcal{C}_{i}^{\prime} & =\left\{\left|\mathcal{V}^{\prime}: \vec{l}, \vec{\nu}\right\rangle \mid I^{\prime}(\vec{l}, \vec{\nu})=i\right\}, \quad i=0, \ldots, M, \\
\mathcal{C}_{i}^{\prime \prime} & =\left\{\left|\mathcal{V}^{\prime \prime}: \vec{l}, \vec{\nu}\right\rangle \mid I^{\prime \prime}(\vec{l}, \vec{\nu})=i\right\}, \quad i=0, \ldots, M
\end{aligned}
$$

By Lemma 1,

$$
\left\{\left|\mathcal{V}^{\prime}: \vec{l}, \vec{\nu}\right\rangle\right\}=\bigcup_{i=0}^{M} \mathcal{C}_{i}^{\prime},
$$

since every $(\vec{l}, \vec{\nu})$ maps to the unique $i$ for which $\omega^{\prime}(\vec{l}, \vec{\nu})=1$,

$$
\mathcal{C}_{i}^{\prime} \bigcap \mathcal{C}_{j}^{\prime}=\emptyset, \quad i \neq j
$$

since no $(\vec{l}, \vec{\nu})$ maps to more than one value of $i$, and similarly

$$
\begin{aligned}
& \left\{\left|\mathcal{V}^{\prime \prime}: \vec{l}, \vec{\nu}\right\rangle\right\}=\bigcup_{i=0}^{M} \mathcal{C}_{i}^{\prime \prime}, \\
& \mathcal{C}_{i}^{\prime \prime} \cap \mathcal{C}_{j}^{\prime \prime}=\emptyset, \quad i \neq j,
\end{aligned}
$$


It is not possible that $\mathcal{C}_{i}^{\prime}$ is empty for some value of $i$, say $\widetilde{i}$. That would imply, using (203) and (207), that there is no $(\vec{l}, \vec{\nu})$ for which $\omega_{i}^{\prime}(\vec{l}, \vec{\nu})$ is nonzero: $\omega_{i}^{\prime}(\vec{l}, \vec{\nu})=0 \quad \forall \vec{l}, \vec{\nu}$. With (193), this implies that $\widehat{Q}_{\widetilde{i}}^{\prime}=0$, contradicting (181). So,

$$
\mathcal{C}_{i}^{\prime} \neq \emptyset, \quad i=0, \ldots, M
$$

and, similarly,

$$
\mathcal{C}_{i}^{\prime \prime} \neq \emptyset, \quad i=0, \ldots, M
$$

By hypothesis,

$$
\sum_{i=0}^{M} \widehat{b}_{i}^{\prime} \otimes \widehat{Q}_{i}^{\prime}=\sum_{i=0}^{M} \widehat{b}_{i}^{\prime \prime} \otimes \widehat{Q}_{i}^{\prime \prime},
$$

Taking the matrix elements of both sides of (215) between $|\mathcal{O}: 0\rangle\left|\mathcal{V}^{\prime}: \vec{l}, \vec{\nu}\right\rangle$ and $|\mathcal{O}: 0\rangle\left|\mathcal{V}^{\prime}: \vec{k}, \vec{\mu}\right\rangle$, and using (8), (175), (183), (190), (191), (198), (199), (203), (204), and Lemma 1,

$$
\left(\beta_{I^{\prime}(\vec{l}, \vec{\nu})}^{\prime}-\beta_{I^{\prime \prime}(\vec{k}, \vec{\mu})}^{\prime \prime}\right)\left\langle\mathcal{V}^{\prime}: \vec{l}, \vec{\mu} \mid \mathcal{V}^{\prime \prime}: \vec{k}, \vec{\mu}\right\rangle=0
$$

$\operatorname{Fix}(\vec{k}, \vec{\mu})=\left(\vec{k}_{1}, \vec{\mu}_{1}\right)$, and define

$$
i_{1}^{\prime}=I^{\prime}\left(\vec{k}_{1}, \vec{\mu}_{1}\right)
$$

There must be some $(\vec{l}, \vec{\nu})=\left(\vec{l}_{1}, \vec{\nu}_{1}\right)$ such that

$$
\left\langle\mathcal{V}^{\prime}: \vec{l}_{1}, \vec{\nu}_{1} \mid \mathcal{V}^{\prime \prime}: \vec{k}_{1}, \vec{\mu}_{1}\right\rangle \neq 0
$$

(If the inner product in (218) vanished for all $\left|\mathcal{V}^{\prime}: \vec{l}_{1}, \vec{\nu}_{1}\right\rangle$, that would imply, with (178), that $\left.\left.\mid \mathcal{V}^{\prime \prime}: \vec{k}_{1}, \vec{\mu}\right)\right\rangle=0$, implying $\left\langle\mathcal{V}^{\prime \prime}: \vec{k}_{1}, \vec{\mu}_{1}\right)\left|\mathcal{V}^{\prime \prime}: \vec{k}_{1}, \vec{\mu}_{1}\right\rangle=0$, contradicting (199).) So,

$$
\left(\beta_{i_{1}^{\prime}}^{\prime}-\beta_{i_{1}^{\prime \prime}}^{\prime \prime}\right)\left\langle\mathcal{V}^{\prime}: \vec{l}_{1}, \vec{\mu}_{1} \mid \mathcal{V}^{\prime \prime}: \vec{k}_{1}, \vec{\mu}_{1}\right\rangle=0
$$

where

$$
i_{1}^{\prime \prime}=I^{\prime \prime}(\vec{k}, \vec{\mu})
$$

implies, with (218),

$$
\beta_{i_{1}^{\prime}}^{\prime}=\beta_{i_{1}^{\prime \prime}}^{\prime \prime}
$$

Consider any other vector $\left|\mathcal{V}^{\prime}: \vec{l}_{1 b}, \vec{\nu}_{1 b}\right\rangle$ which has nonzero inner product with $\left|\mathcal{V}^{\prime \prime}: \vec{k}_{1}, \vec{\mu}_{1}\right\rangle$,

$$
\left\langle\mathcal{V}^{\prime}: \vec{l}_{1 b}, \vec{\nu}_{1 b} \mid \mathcal{V}^{\prime \prime}: \vec{k}_{1}, \vec{\mu}_{1}\right\rangle \neq 0
$$

Using (216), (217) and (222)

$$
\beta_{I^{\prime}\left(\vec{l}_{1 b}, \vec{\nu}_{1 b}\right)}^{\prime}=\beta_{i_{1}^{\prime \prime}}^{\prime \prime}
$$

From (221) and (223),

$$
\beta_{I^{\prime}\left(\vec{l}_{1 b}, \vec{\nu}_{1 b}\right)}^{\prime}=\beta_{i_{1}^{\prime}}^{\prime}
$$

which, with the nondegeneracy condition (176), implies

$$
I^{\prime}\left(\vec{l}_{1 b}, \vec{\nu}_{1 b}\right)=i_{1}^{\prime}
$$


That is, all vectors $\left|\mathcal{V}^{\prime}: \vec{l}, \vec{\nu}\right\rangle$ which have nonzero inner product with $\left|\mathcal{V}^{\prime \prime}: \vec{k}_{1}, \vec{\nu}_{1}\right\rangle$ are in $\mathcal{C}_{i_{1}^{\prime}}^{\prime}$. Repeating the same argument with any other vector in $\mathcal{C}_{i_{1}^{\prime \prime}}^{\prime \prime}$ instead of $\left|\mathcal{V}^{\prime \prime}: \vec{k}_{1}, \vec{\nu}_{1}\right\rangle$ leads to the same conclusion. So,

$$
\left\langle\mathcal{V}^{\prime}: \vec{l}, \vec{\nu} \mid \mathcal{V}^{\prime \prime}: \vec{k}, \vec{\mu}\right\rangle=\delta_{i_{1}^{\prime}, I^{\prime}(\vec{l}, \vec{\nu})}\left\langle\mathcal{V}^{\prime}: \vec{l}, \vec{\nu} \mid \mathcal{V}^{\prime \prime}: \vec{k}, \vec{\mu}\right\rangle, \quad\left|\mathcal{V}^{\prime \prime}: \vec{k}, \vec{\mu}\right\rangle \in \mathcal{C}_{i_{1}^{\prime \prime}}^{\prime \prime}
$$

Starting again but this time with a vector in $\mathcal{C}_{i_{2}^{\prime \prime}}^{\prime \prime}$, where $i_{2}^{\prime \prime} \neq i_{1}^{\prime \prime}$, we obtain

$$
\left\langle\mathcal{V}^{\prime}: \vec{l}, \vec{\nu} \mid \mathcal{V}^{\prime \prime}: \vec{k}, \vec{\mu}\right\rangle=\delta_{i_{2}^{\prime}, I^{\prime}(\vec{l}, \vec{\nu})}\left\langle\mathcal{V}^{\prime}: \vec{l}, \vec{\nu} \mid \mathcal{V}^{\prime \prime}: \vec{k}, \vec{\mu}\right\rangle, \quad\left|\mathcal{V}^{\prime \prime}: \vec{k}, \vec{\mu}\right\rangle \in \mathcal{C}_{i_{2}^{\prime \prime}}^{\prime \prime}
$$

for some $i_{2}^{\prime}$. It's not possible that $i_{2}^{\prime}=i_{1}^{\prime}$. If $\left|\mathcal{V}^{\prime}: \vec{l}, \vec{\nu}\right\rangle \in \mathcal{C}_{i_{2}^{\prime}}^{\prime},\left|\mathcal{V}^{\prime \prime}: \vec{k}, \vec{\mu}\right\rangle \in \mathcal{C}_{i_{2}^{\prime \prime}}^{\prime \prime}$, and $\left\langle\mathcal{V}^{\prime}: \vec{l}, \vec{\nu} \mid \mathcal{V}^{\prime \prime}: \vec{k}, \vec{\mu}\right\rangle \neq 0$, then we conclude from (216) that

$$
\beta_{i_{2}^{\prime}}^{\prime}=\beta_{i_{2}^{\prime \prime}}^{\prime \prime}
$$

If $i_{2}^{\prime}=i_{1}^{\prime}$ then (221) and (228) imply

$$
\beta_{i_{1}^{\prime \prime}}^{\prime \prime}=\beta_{i_{2}^{\prime \prime}}^{\prime \prime}
$$

But we have chosen $i_{2}^{\prime \prime}$ to be unequal to $i_{1}^{\prime \prime}$ so (229) contradicts the nondegeneracy condition (184).

By virtue of (213) and (214) we can continue in this manner to obtain

$$
\begin{aligned}
\left\langle\mathcal{V}^{\prime}: \vec{l}, \vec{\nu} \mid \mathcal{V}^{\prime \prime}: \vec{k}, \vec{\mu}\right\rangle & =\delta_{i_{1}^{\prime}, I^{\prime}(\vec{l}, \vec{\nu})}\left\langle\mathcal{V}^{\prime}: \vec{l}, \vec{\nu} \mid \mathcal{V}^{\prime \prime}: \vec{k}, \vec{\mu}\right\rangle, \quad\left|\mathcal{V}^{\prime \prime}: \vec{k}, \vec{\mu}\right\rangle \in \mathcal{C}_{i_{1}^{\prime \prime}}^{\prime \prime} \\
& \vdots \\
\left\langle\mathcal{V}^{\prime}: \vec{l}, \vec{\nu} \mid \mathcal{V}^{\prime \prime}: \vec{k}, \vec{\mu}\right\rangle & =\delta_{i_{M+1}^{\prime}, I^{\prime}(\vec{l}, \vec{\nu})}\left\langle\mathcal{V}^{\prime}: \vec{l}, \vec{\nu} \mid \mathcal{V}^{\prime \prime}: \vec{k}, \vec{\mu}\right\rangle, \quad\left|\mathcal{V}^{\prime \prime}: \vec{k}, \vec{\mu}\right\rangle \in \mathcal{C}_{i_{M+1}^{\prime \prime}}^{\prime \prime}
\end{aligned}
$$

where

$$
\begin{gathered}
i_{j}^{\prime} \neq i_{k}^{\prime} \quad \text { for } j, k=1, \ldots, M+1, \quad j \neq k \\
i_{j}^{\prime \prime} \neq i_{k}^{\prime \prime} \quad \text { for } j, k=1, \ldots, M+1, \quad j \neq k \\
0 \leq i_{j}^{\prime}, i_{k}^{\prime \prime}, \leq M, \quad j, k=1, \ldots, M+1 .
\end{gathered}
$$

Defining the mapping $\pi(i)$ by

$$
i_{1}^{\prime}=\pi\left(i_{1}^{\prime \prime}\right), i_{2}^{\prime}=\pi\left(i_{2}^{\prime \prime}\right), \ldots, i_{M+1}^{\prime}=\pi\left(i_{M+1}^{\prime \prime}\right),
$$

eqs. (231)-(233) tell us that $\pi(i)$ is a permutation of $0, \ldots, M$. Using (234) we can write (230) as

$$
\begin{aligned}
\left\langle\mathcal{V}^{\prime}: \vec{l}, \vec{\nu} \mid \mathcal{V}^{\prime \prime}: \vec{k}, \vec{\mu}\right\rangle & =\delta_{\pi\left(i_{1}^{\prime \prime}\right), I^{\prime}(\vec{l}, \vec{\nu})}\left\langle\mathcal{V}^{\prime}: \vec{l}, \vec{\nu} \mid \mathcal{V}^{\prime \prime}: \vec{k}, \vec{\mu}\right\rangle, \quad\left|\mathcal{V}^{\prime \prime}: \vec{k}, \vec{\mu}\right\rangle \in \mathcal{C}_{i_{1}^{\prime \prime}}^{\prime \prime} \\
& \vdots \\
\left\langle\mathcal{V}^{\prime}: \vec{l}, \vec{\nu} \mid \mathcal{V}^{\prime \prime}: \vec{k}, \vec{\mu}\right\rangle & =\delta_{\pi\left(i_{M+1}^{\prime \prime}\right), I^{\prime}(\vec{l}, \vec{\nu})}\left\langle\mathcal{V}^{\prime}: \vec{l}, \vec{\nu} \mid \mathcal{V}^{\prime \prime}: \vec{k}, \vec{\mu}\right\rangle, \quad\left|\mathcal{V}^{\prime \prime}: \vec{k}, \vec{\mu}\right\rangle \in \mathcal{C}_{i_{M+1}^{\prime \prime}}^{\prime \prime}
\end{aligned}
$$

But from (208),

$$
\left|\mathcal{V}^{\prime \prime}: \vec{k}, \vec{\mu}\right\rangle \in \mathcal{C}_{i}^{\prime \prime} \Rightarrow i=I^{\prime \prime}(\vec{k}, \vec{\mu}), \quad i=0, \ldots, M
$$


so, keeping in mind (211), the equations (235) can be written as

$$
\left\langle\mathcal{V}^{\prime}: \vec{l}, \vec{\nu} \mid \mathcal{V}^{\prime \prime}: \vec{k}, \vec{\mu}\right\rangle=\delta_{\pi\left(I^{\prime \prime}(\vec{k}, \vec{\mu})\right), I^{\prime}(\vec{l}, \vec{\nu})}\left\langle\mathcal{V}^{\prime}: \vec{l}, \vec{\nu} \mid \mathcal{V}^{\prime \prime}: \vec{k}, \vec{\mu}\right\rangle
$$

Using (178) and (201),

$$
\begin{aligned}
\widehat{Q}_{i}^{\prime \prime}= & \sum_{\vec{l}, \vec{l}^{\prime}, \vec{k}} \int d \vec{\nu} d \vec{\nu}^{\prime} d \vec{\mu} \\
& \left|\mathcal{V}^{\prime}: \vec{l}, \vec{\nu}\right\rangle\left\langle\mathcal{V}^{\prime}: \vec{l}, \vec{\nu} \mid \mathcal{V}^{\prime \prime}: \vec{k}, \vec{\mu}\right\rangle \omega_{i}^{\prime \prime}(\vec{k}, \vec{\mu})\left\langle\mathcal{V}^{\prime \prime}: \vec{k}, \vec{\mu} \mid \mathcal{V}^{\prime}: \overrightarrow{l^{\prime}}, \vec{\nu}^{\prime}\right\rangle\left\langle\mathcal{V}^{\prime}: \overrightarrow{l^{\prime}}, \vec{\nu}^{\prime}\right| \\
= & \sum_{\vec{l}, \vec{l}^{\prime}, \vec{k}} \int d \vec{\nu} d \vec{\nu}^{\prime} d \vec{\mu} \delta_{\left.\pi\left(I^{\prime \prime}(\vec{k}, \vec{\mu})\right), I^{\prime}(\vec{l}, \vec{\nu})\right)} \delta_{\left.\pi\left(I^{\prime \prime}(\vec{k}, \vec{\mu})\right), I^{\prime}\left(\overrightarrow{l^{\prime}}, \vec{\nu}^{\prime}\right)\right)} \\
& \left|\mathcal{V}^{\prime}: \vec{l}, \vec{\nu}\right\rangle\left\langle\mathcal{V}^{\prime}: \vec{l}, \vec{\nu} \mid \mathcal{V}^{\prime \prime}: \vec{k}, \vec{\mu}\right\rangle \omega_{i}^{\prime \prime}(\vec{k}, \vec{\mu})\left\langle\mathcal{V}^{\prime \prime}: \vec{k}, \vec{\mu} \mid \mathcal{V}^{\prime}: \overrightarrow{l^{\prime}}, \vec{\nu}^{\prime}\right\rangle\left\langle\mathcal{V}^{\prime}: \overrightarrow{l^{\prime}}, \vec{\nu}^{\prime}\right| \\
= & \sum_{\vec{l}, \overrightarrow{l^{\prime}}, \vec{k}} \int d \vec{\nu} d \vec{\nu}^{\prime} d \vec{\mu} \delta_{\left.\pi\left(I^{\prime \prime}(\vec{k}, \vec{\mu})\right), I^{\prime}(\vec{l}, \vec{\nu})\right)} \delta_{\left.\pi\left(I^{\prime \prime}(\vec{k}, \vec{\mu})\right), I^{\prime}\left(\vec{l}, \vec{\nu}^{\prime}\right)\right)} \delta_{i, I^{\prime \prime}(\vec{k}, \vec{\mu})} \\
& \left|\mathcal{V}^{\prime}: \vec{l}, \vec{\nu}\right\rangle\left\langle\mathcal{V}^{\prime}: \vec{l}, \vec{\nu} \mid \mathcal{V}^{\prime \prime}: \vec{k}, \vec{\mu}\right\rangle\left\langle\mathcal{V}^{\prime \prime}: \vec{k}, \vec{\mu} \mid \mathcal{V}^{\prime}: \overrightarrow{l^{\prime}}, \vec{\nu}^{\prime}\right\rangle\left\langle\mathcal{V}^{\prime}: \vec{l}^{\prime}, \vec{\nu}^{\prime}\right|
\end{aligned}
$$

using (237) and its complex conjugate, and then (206). So,

$$
\begin{aligned}
\widehat{Q}_{i}^{\prime \prime}= & \sum_{\vec{l}, \vec{l}^{\prime}, \vec{k}} \int d \vec{\nu} d \vec{\nu}^{\prime} d \vec{\mu} \delta_{\left.\pi(i), I^{\prime}(\vec{l} \vec{\nu})\right)} \delta_{\left.\pi(i), I^{\prime}\left(\vec{l}^{\prime} \vec{\nu}^{\prime}\right)\right)} \delta_{i, I^{\prime \prime}(\vec{k}, \vec{\mu})} \\
& \left|\mathcal{V}^{\prime}: \vec{l}, \vec{\nu}\right\rangle\left\langle\mathcal{V}^{\prime}: \vec{l}, \vec{\nu} \mid \mathcal{V}^{\prime \prime}: \vec{k}, \vec{\mu}\right\rangle\left\langle\mathcal{V}^{\prime \prime}: \vec{k}, \vec{\mu} \mid \mathcal{V}^{\prime}: \overrightarrow{l^{\prime}}, \vec{\nu}^{\prime}\right\rangle\left\langle\mathcal{V}^{\prime}: \vec{l}^{\prime}, \vec{\nu}^{\prime}\right| .
\end{aligned}
$$

Considering the first delta function in (239), we see that the summand/integrand is only nonzero for values of $(\vec{l}, \vec{\nu})$ such that

$$
I^{\prime}(\vec{l}, \vec{\nu})=\pi(i)
$$

However, (237) shows that, if (240) holds, $\left\langle\mathcal{V}^{\prime}: \vec{l}, \vec{\nu} \mid \mathcal{V}^{\prime \prime}: \vec{k}, \vec{\mu}\right\rangle$ is only nonzero if

$$
\pi\left(I^{\prime \prime}(\vec{k}, \vec{\mu})\right)=\pi(i)
$$

or

$$
I^{\prime \prime}(\vec{k}, \vec{\mu})=i
$$

since $\pi(i)$ is a permutation. So the third delta function in (239) is superfluous:

$$
\begin{aligned}
\widehat{Q}_{i}^{\prime \prime}= & \sum_{\vec{l}, \overrightarrow{l^{\prime}}, \vec{k}} \int d \vec{\nu} d \vec{\nu}^{\prime} d \vec{\mu} \delta_{\left.\pi(i), I^{\prime}(\vec{l} \vec{\nu})\right)} \delta_{\left.\pi(i), I^{\prime}\left(\overrightarrow{l^{\prime}} \vec{\nu}^{\prime}\right)\right)} \\
& \left|\mathcal{V}^{\prime}: \vec{l}, \vec{\nu}\right\rangle\left\langle\mathcal{V}^{\prime}: \vec{l}, \vec{\nu} \mid \mathcal{V}^{\prime \prime}: \vec{k}, \vec{\mu}\right\rangle\left\langle\mathcal{V}^{\prime \prime}: \vec{k}, \vec{\mu} \mid \mathcal{V}^{\prime}: \overrightarrow{l^{\prime}}, \vec{\nu}^{\prime}\right\rangle\left\langle\mathcal{V}^{\prime}: \overrightarrow{l^{\prime}}, \vec{\nu}^{\prime}\right| \\
= & \sum_{\vec{l}} \int d \vec{\nu}\left|\mathcal{V}^{\prime}: \vec{l}, \vec{\nu}\right\rangle \omega_{\pi(i)}^{\prime}(\vec{l}, \vec{\nu})\left\langle\mathcal{V}^{\prime}: \vec{l}, \vec{\nu}\right|
\end{aligned}
$$

using (191), (200) and (206). Comparing the rightmost members of (193) and (243), we conclude

$$
\widehat{Q}_{i}^{\prime \prime}=\widehat{Q}_{\pi(i)}^{\prime}, \quad i=0, \ldots, M
$$


as claimed.

Using (244) in (215)

$$
\sum_{i=1}^{M} \widehat{b}_{i}^{\prime} \otimes \widehat{Q}_{i}^{\prime}=\sum_{i=1}^{M} \widehat{b}_{i}^{\prime \prime} \otimes \widehat{Q}_{\pi(i)}^{\prime},
$$

Taking the matrix elements of both sides of (245) between arbitrary vectors $\left|\mathcal{V}^{\prime}: \vec{l}, \vec{\nu}\right\rangle$, $\left|\mathcal{V}^{\prime}: \vec{l}^{\prime}, \vec{\nu}^{\prime}\right\rangle$, using (177), (198) and (205), summing over $\vec{l}^{\prime}$ and integrating over $\vec{\nu}^{\prime}$,

$$
\sum_{i=0}^{M}\left(\widehat{b}_{\pi(i)}^{\prime}-\widehat{b}_{i}^{\prime \prime}\right) \delta_{\pi(i), I^{\prime}(\vec{l}, \vec{\nu})}=0 .
$$

or

$$
\widehat{b}_{I^{\prime}(\vec{l}, \vec{\nu})}^{\prime}-\widehat{b}_{\pi^{-1}\left(I^{\prime}(\vec{l}, \vec{\nu})\right)}^{\prime \prime}=0 .
$$

Since $|\mathcal{V}: \vec{l}, \vec{\nu}\rangle$ is arbitrary and $\mathcal{C}_{i}^{\prime} \neq \emptyset$ we can conclude that

$$
\widehat{b}_{i}^{\prime}-\widehat{b}_{\pi^{-1}(i)}^{\prime \prime}=0, \quad i=0, \ldots, M,
$$

or

$$
\widehat{b}_{i}^{\prime \prime}=\widehat{b}_{\pi(i)}^{\prime}, \quad i=0, \ldots, M
$$

as claimed.

\section{References}

[1] H. Everett III, " 'Relative state' formulation of quantum mechanics, " Rev. Mod. Phys. 29, 454-462 (1957). Reprinted in Ref. 2.

[2] B. S. DeWitt and N. Graham, eds., The Many Worlds Interpretation of Quantum Mechanics (Princeton University, Princeton, NJ, 1973).

[3] M. C. Price, "The Everett FAQ," http://www.hedweb.com/manworld.htm (1995).

[4] L. Vaidman, "Many-worlds interpretation of quantum mechanics," in Stanford Encyclopedia of Philosophy (Summer 2002 Edition), E. N. Zalta, ed., http://plato.stanford.edu/archives/sum2002/entries/qm-manyworlds.

[5] W. H. Zurek, "Pointer basis of quantum apparatus: Into what mixture does the wave packet collapse?," Phys. Rev. D24, 1516-1525 (1981).

[6] W. H. Zurek, "Environment-induced superselection rules," Phys. Rev. D26, 1862-1880 (1982).

[7] W. H. Zurek, "Decoherence and the transition from quantum to classical-revisited," Los Alamos Science, no. 27, 2-25 (2002); quant-ph/0306072.

[8] J. Barrett, "Everett's relative-state formulation of quantum mechanics", in Stanford Encyclopedia of Philosophy (Spring 2003 Edition), E. N. Zalta, ed., http://plato.stanford.edu/archives/spr2003/entries/qm-everett/. 
[9] W. H. Zurek, "Quantum Darwinism and envariance," quant-ph/0308163 (2003).

[10] M. A. Rubin, "There is no basis ambiguity in Everett quantum mechanics," Found. Phys. Lett. 17, 323-341 (2004) quant-ph/0310186

[11] B. S. DeWitt, "The many-universes interpretation of quantum mechanics," in B. d'Espagnat, ed., Proceedings of the International School of Physics "Enrico Fermi" Course IL: Foundations of Quantum Mechanics (Academic Press, New York, 1972). Reprinted in Ref. 2.

[12] B. DeWitt, "The quantum mechanics of isolated systems," Int. J. Mod. Phys. A13, 1881-1916 (1998).

[13] M. Schlosshauer, "Decoherence, the measurement problem, and interpretations of quantum mechanics," Rev. Mod. Phys 76, 1267-1305 (2004); quant-ph/0312059.

[14] H. P. Stapp, "The basis problem in many-worlds theories," Can. J. Phys., 86, 10431052 (2002); quant-ph/0110148.

[15] M. A. Rubin, "Locality in the Everett interpretation of Heisenberg-picture quantum mechanics," Found. Phys. Lett. 14, 301-322 (2001); quant-ph/0103079.

[16] M. A. Rubin, "Relative frequency and probability in the Everett interpretation of Heisenberg-picture quantum mechanics," Found. Phys. 33, 379-405 (2003); quant-ph/0209055.

[17] M. A. Rubin, "Locality in the Everett interpretation of quantum field theory," Found. Phys. 32, 1495-1523 (2002); quant-ph/0204024.

[18] B. d'Espagnat, Conceptual Foundations of Quantum Mechanics, 2nd edn. (W. A. Benjamin, Reading, MA, 1976).

[19] F. J. Tipler, "Does quantum nonlocality exist? Bell's theorem and the many-worlds interpretation," quant-ph/0003146.

[20] D. Deutsch, and P. Hayden, "Information flow in entangled quantum systems," Proc. R. Soc. Lond. A456, 1759-1774 (2000); quant-ph/9906007.

[21] H. Cramér, Mathematical Methods of Statistics (Princeton University, Princeton, NJ, 1946).

[22] G. Pólya, Mathematics and Plausible Reasoning, Vol. II, 2nd. ed. (Princeton University, Princeton, NJ, 1968).

[23] J. J. Halliwell, "Somewhere in the universe: Where is the information stored when histories decohere?," Phys. Rev. D 60, 105031 (1999) quant-ph/9902008.

[24] L. E. Ballentine, Quantum Mechanics: A Modern Development (World Scientific, Singapore, 1998). 
[25] M. Rosenlicht, Introduction to Analysis (Dover, Mineola, NY 1986).

[26] B. d'Espagnat, "A note on measurement," Physics Letters A, 282, 133-137 (2001).

[27] M. K. Gaillard, P. D. Grannis and F. J. Sciulli, "The standard model of particle physics," Rev. Mod. Phys. 71, S96-S111 (1999).

[28] L. Brown, Quantum Field Theory (Cambridge University, Cambridge, 1992).

[29] S. Weinberg, The Quantum Theory of Fields: Vol. I, Foundations (Cambridge University, Cambridge, 1995).

[30] E. Joos, "Comment on 'Unified dynamics for microscopic and macroscopic systems'," Phys Rev. D 36, 3285-3286 (1987).

[31] D. N. Page, "Probabilities don't matter," in M. Keiser and R. T. Jantsen, eds., Proceedings of the 7th Marcel Grossmann Meeting on General Relativity, (World Scientific, Singapore, 1995); gr-qc/9411004.

[32] D. N. Page,"Sensible quantum mechanics: Are only perceptions probabilistic?," quant-ph/9506010.

[33] D. N. Page, "Sensible quantum mechanics: Are probabilities only in the mind?," Int. J. Mod. Phys. D5, 583-596 (1996); gr-qc/9507042.

[34] E. R. Kandel, J. H. Schwartz, and T. M. Jessell, Principles of Neural Science, 4th edn. (McGraw-Hill, New York, 2000).

[35] A. Shimony, "Comments on Legett's 'macroscopic realism'," in R. A. Healey and G. Hellman, eds., Quantum Measurement: Beyond Paradox (University of Minnesota, Minneapolis, 1998).

[36] P. St. Hilaire, D. Bierman and S. Hameroff, "Quantum states in the retina," http://www.quantumconsciousness.org/views/QuantumStatesRetina.html (2002).

[37] M. Schlosshauer, "Experimental motivation and empirical consistency in minimal nocollapse quantum mechanics," Ann. Phys. 321, 112-149 (2006); quant-ph/0506199

[38] F. T. Thaheld, "Does consciousness really collapse the wave function?: A possible objective biophysical resolution to the measurement problem," BioSystems 81, 113124 (2005); quant-ph/050942.

[39] F. T. Thaheld, "Comment on 'Experimental motivation and empirical consistency in minimal no-collapse quantum mechanics'," quant-ph/0602190.

[40] P. van Esch, "On the Everett programme and the Born rule," quant-ph/0505059.

[41] J. R. Friedman, V. Patel, W. Chen, S. K. Tolpygo and J. E. Lukens, "Detection of a Schrödinger's cat state in an rf-SQUID," Nature 406, 43-46 (2000); cond-mat/0004293. 
[42] J. J. Halliwell, "Some recent developments in the decoherent histories approach to quantum theory," in H.-T. Elze, ed., Decoherence and Entropy in Complex Systems: Selected Lectures from DICE 2002 (Springer, Berlin, 2004); quant-ph/0301117.

[43] E. Joos and H. D. Zeh, "The emergence of classical properties through interaction with the environment," Z. Phys. B59, 223-243 (1985).

[44] K. Hornberger and J. E. Sipe, "Collisional decoherence reexamined," Phys. Rev. A 68, 012105 (2003); quant-ph/0303094.

[45] K. Hornberger, S. Uttenhaler, B. Brezger, L. Hackermueller, M. Arndt and A. Zeilinger, "Collisional decoherence observed in matter wave interferometry," Phys. Rev. Lett. 90, 160401 (2003); quant-ph/0303093.

[46] L. Hackermueller, K. Hornberger, B. Brezger, A. Zeilinger and M. Arndt, "Decoherence in a Talbot-Lau interferometer: the influence of molecular scattering," Appl. Phys. B 77, 781-787 (2003); quant-ph/0307238.

[47] L. Hackermueller, S. Uttenthaler, K. Hornberger, E. Reiger, B. Brezger, A. Zeilinger, M. Arndt, "The wave nature of biomolecules and fluorofullerenes," Phys. Rev. Lett. 91, 090408 (2003) quant-ph/0309016. 